\title{
Whole-Transcriptome Analysis of Serum L1CAM- Captured Extracellular Vesicles Reveals Neural And Glycosylation Changes In Autism Spectrum Disorder
}

\author{
Yannan Qin \\ Xi'an Jiaotong University Health Science Center \\ Li Cao \\ Xi'an Jiaotong University Health Science Center \\ Haiqing Zhang \\ Xi'an Jiaotong University

\section{Shuang Cai} \\ Xi'an Jiaotong University Health Science Center \\ Jinyuan Zhang \\ Xi'an Jiaotong University Health Science Center
}

\section{Bo Guo}

Xi'an Jiaotong University Health Science Center

\section{Fei Wu}

Xi'an Jiaotong University Health Science Center

\section{Lingyu Zhao}

Xi'an Jiaotong University Health Science Center

\section{Wen Li}

Xi'an Jiaotong University Health Science Center

Lei $\mathrm{Ni}$

Xi'an Jiaotong University Health Science Center

Liying Liu

Biomedical Experimental Center of Xi'an Jiaotong University

\section{Xiaofei Wang}

Biomedical Experimental Center of Xi'an Jiaotong University

\section{Yanni Chen}

Xi'an Jiaotong University

Chen Huang ( $\nabla$ hchen@mail.xjtu.edu.cn )

Xi'an Jiaotong University Health Science Center https://orcid.org/0000-0002-2407-9716 


\section{Research Article}

Keywords: Autism spectrum disorder, L1CAM-captured extracellular vesicles, transcriptome, biomarker, neuron-related network, glycan-related network

Posted Date: December 20th, 2021

DOI: https://doi.org/10.21203/rs.3.rs-1143525/v1

License: (c) (i) This work is licensed under a Creative Commons Attribution 4.0 International License.

Read Full License

Version of Record: A version of this preprint was published at Journal of Molecular Neuroscience on April 12th, 2022. See the published version at https://doi.org/10.1007/s12031-022-01994-z. 


\section{Abstract}

The pathophysiology of autistic spectrum disorder (ASD) is not fully understood and there are no diagnostic or predictive biomarkers. Extracellular vesicles (EVs) are cell-derived nano-sized vesicles, carrying nucleic acids, proteins, lipids and other bioactive substances. As reported, serum neural cell adhesion molecule L1 (L1CAM)-captured EVs (LCEVs) can provide reliable biomarkers for neurological diseases; however, little is known about the LCEVs in children with ASD. In this study, serum samples were collected from 100 ASD children and 60 age-matched typically developed (TD) children. LCEVs were isolated and characterized meticulously. Whole-transcriptome of LCEVs was analyzed by IncRNA microarray and RNA-Sequencing. All raw data was submitted on GEO Profiles, and GEO accession numbers is GSE186493. RNAs expressed differently in LCEVs from ASD sera vs. TD sera were screened, analyzed, and further validated. A total of 1418 mRNAs, 1745 IncRNAs and 11 miRNAs were differentially expressed, and most of them were down-regulated in ASD. Most RNAs were involved in neuron- and glycan-related networks implicated in ASD. The levels of EDNRA, SLC17A6, HTR3A, OSTC, TMEM165, PC5p-139289_26, and hsa-miR-193a-5p changed significantly in ASD. In conclusion, whole-transcriptome analysis of serum LCEVs reveals neural and glycosylation changes in ASD, which may help detect predictive biomarkers and molecular mechanisms of ASD, and provide reference for diagnoses and therapeutic management of the disease.

\section{Introduction}

Autism spectrum disorder (ASD) refers to a set of early-appearing social communication deficits and repetitive sensory-motor behaviors associated with a strong genetic component as well as other causes (Lord, Elsabbagh, Baird, \& Veenstra-Vanderweele, 2018). The prevalence of ASD is region-specific, varying from 0.9 case out of 1000 children in India to 1 case within 59children in the US (Hyman et al., 2020). In China, the prevalence is now around $1 \%$ (Sun et al., 2019). Screening of high-risk population is the first step toward early detection and diagnosis of ASD, thereby impacting the likelihood of patients accessing early intervention and, importantly, improving long-term outcomes. Currently, clinical diagnosis of ASD is still based on the fifth edition of the Diagnostic and Statistical Manual of Mental Disorders (DSM-V) (American Psychiatric Association, 2013). Such diagnosis is focused on assessing patients' behaviors, but it lacks quantizable indicators and cannot accurately identify mild or non-typical autism or autism in very young patients(Weiss et al., 2009). There is a need to find useful and reliable biomarkers to facilitate the diagnosis of autism.

In the past decade, searching for genetic biomarkers has been a hot spot in ASD research. Numerous related genes have been reported, including NRXN1, SHANK3, SHANK2, MECP2, SNC2A, CHD8, DYRKIA, POG2, GRIN2B, KATNAL2, NLGN3, NLGN4, CNTN4, CDH10, CDH9, and SEMA5A (Kramer, Beer, Bode, \& Winter, 2020; Sanders et al., 2012; Wakefield, 2016). Unfortunately, only 10-38\% of ASD cases have been reported with known genetic deficits (Lin, Liu, \& Roeder, 2021; Vorstman et al., 2017). In recent years, blood/serum biomarkers have drawn much attention due to their accessibility, low cost and rapid detection. In our previous studies, we identified four candidate peptides (SerpinA5-A, ApoC1-A, FABP1-A 
and PF4-A) (Yang et al., 2018) and a2-3-linked sialylation of apolipoprotein D (APOD) (Qin et al., 2017)as potential biomarkers for ASD. A recent study also reported that SLC25A12, LIMK1 and RARS might serve as potential blood protein biomarkers for ASD (Yao et al., 2021). However, the reported potential biomarkers cannot specifically reflect the abnormality of brain neurons preferentially affected in autism and reveal the dysregulation of specific genes in neurons correlated with clinical severity (Jin et al., 2020; Velmeshev et al., 2019).

Extracellular vesicles (EVs) are a heterogeneous group of nano-sized, cell-derived membranous structures comprising exosomes, microvesicles and others. They contain components from the cells that release them, such as proteins, lipids, nucleic acids and glycoconjugates. In the central nervous system, almost all types of cells secrete EVs, which mediate neuron-glial cell communication, promote neuronal repair and growth, and promote the progression of glioblastoma and neurological diseases(Fayazi, Sheykhhasan, Asl, \& Najafi, 2021). EVs are structurally stable and protect the "biological cargo" they carry from degradation and denaturation in the extracellular environment. Compared with biological fluids, such as cerebrospinal fluid, blood or urine, EVs can provide more reliable and accurate biomarkers for neurological diseases(Filippone \& Pratico, 2021; Wang et al., 2021). More importantly, they can cross the blood-brain barrier and have low immunogenicity(Saint-Pol, Gosselet, Duban-Deweer, Pottiez, \& Karamanos, 2020). Overall, EVs are a promising source for biomarkers and ideal vehicles for drug delivery, and might be used in the diagnosis and treatment of neurological diseases(Andjus et al., 2020; Hill, 2019). Currently, it is found that secreted EVs increase in the serum of ASD children and contain IL-1 $\beta$ that stimulates secretion of human microglia cells(Tsilioni \& Theoharides, 2018). As has been reported, mesenchymal stem cell-derived exosomes can improve autism-like behavior in BTBR mice and may be a cell-free therapeutic tool for ASD (Alessio et al., 2020; Perets, Oron, Herman, Elliott, \& Offen, 2020). These findings uncover important roles of EVs, suggesting the necessity of characterizing the detailed molecular status of brain-derived EVs in ASD.

Recent studies have shown that the surface of EVs derived from neurons carries neural cell adhesion molecule L1 (L1CAM) that can be utilized to isolate the specific EVs from serum/plasma(Goetzl, Abner, Jicha, Kapogiannis, \& Schwartz, 2018; Goetzl, Peltz, Mustapic, Kapogiannis, \& Yaffe, 2020; Nogueras-Ortiz et al., 2020). Proteins in L1CAM captured exosomes can reflect brain injury, progression from acute mild traumatic brain injury to chronic traumatic brain disease, cognitive dysfunction caused by HIV infection, and neurological abnormalities such as Alzheimer's disease(Goetzl et al., 2018; Goetzl et al., 2020). Blood neuron-derived EVs (using anti-L1CAM antibody) from Alzheimer's disease patients effect complementmediated neurotoxicity(Nogueras-Ortiz et al., 2020). However, the expression of proteins or RNAs in L1CAM captured EVs (LCEVs) of ASD children is rarely reported.

It is well known that EVs miRNAs can be used as potential diagnostic and prognostic biomarkers as well as therapeutic tools for a variety of neuropsychiatric diseases, such as dementia, Alzheimer's disease, depression, and schizophrenia (Fries \& Quevedo, 2018; Hill, 2019). In light of this, the present study was conducted to examine the expression of RNAs in LCEVs from children with ASD and to reveal the potential biomarkers and possible mechanisms of the disease. We collected serum samples from 100 
ASD children and 60 age-matched typically developed (TD) children. LCEVs were isolated using L1CAM antibody mediated immunosorbent assay and characterized by nanoparticle tracking analysis, transmission electron microscopy and western blot. Whole-transcriptome of the LCEVs was analyzed by IncRNA microarray and RNA-Sequencing. In brief, a total of 1418 mRNAs, 1745 IncRNAs and 11 miRNAs were found differentially expressed. Most of these RNAs were down-regulated in ASD and enriched in neuron-related and glycan-related networks associated with ASD. Levels of some potential biomarkers were found significantly changed in ASD.

\section{Methods}

\section{Study approval}

Approval for this study was obtained from the Ethics Committee of Xi'an Jiaotong University (Xi'an, China). A parent of each participant signed a written informed consent. The experiments were carried out in accordance with the ethical guidelines of the Declaration of Helsinki.

\section{Subjects}

The study enrolled 100 children with ASD (2.5- 6 years of age; 90 males) and 60 age-matched TD children (54 males) as control. The ASD children were recruited from Xi'an Children's Hospital, Xi'an, China. The healthy children were recruited from the same region to minimize the influence of different environments. The ASD children were examined by a developmental behavioral pediatrician and a pediatric neurologist or psychiatrist. All the consultants agreed on ASD diagnosis according to the DSM-V criteria. Children with tuberous sclerosis complex, Rett syndrome, Prader Willi syndrome, or Angelman syndrome were excluded. All the participants were screened via a parental interview for current and past physical illnesses. Those who had any type of infection or disease within two weeks before the time of examination were excluded. ASD was evaluated with the autism diagnostic observation schedule (Table 1). 
Table 1

Basic characteristics of the participants

\begin{tabular}{|llll|}
\hline \multicolumn{1}{|c}{ ASD } & TD & p-Value \\
\hline $\mathrm{N}$ & 100 & 60 & - \\
\hline Males, $(\mathrm{n}) \%$ & $90(90 \%)$ & $54(90 \%)$ & - \\
\hline Age, years ${ }^{\mathrm{a}}$ & $3.5(2.5-5.5)$ & $3.5(2.5-6.0)$ & 0.980 \\
\hline Autism diagnostic observation schedule (ADOS) ${ }^{\mathrm{b}}$ & & \\
\hline Language and communication & $5.14 \pm 2.78$ & \\
\hline Reciprocal social interaction & $11.36 \pm 3.23$ & \\
\hline Stereotyped behaviors and restricted interests & $1.59 \pm 1.23$ & Severe: $19 \%$ \\
\hline Total score & & $16.64 \pm 4.39$ & Moderate: $64 \%$ \\
\hline Severity degree & Mild: $17 \%$ & & \\
\hline a Median (range). ${ }^{\mathrm{b}}$ Average \pm SD & & & \\
\hline
\end{tabular}

\section{Collection and preparation of serum samples}

Venous blood samples were collected by a pediatric nurse. The blood was allowed to clot at room temperature for $30 \mathrm{~min}$, and the clot was then removed by centrifuging at 1,500×g for 10 minutes. The resulting supernatant is immediately transferred to a clean polypropylene tube, and EDTA-free inhibitor cocktail (Halt protease inhibitor; Thermo Scientific Pierce Protein Research Products, Rockford, IL, USA) was added at a concentration of $10 \mu \mathrm{L} / \mathrm{mL}$ serum. The obtained serum was aliquot into small portions and was immediately frozen on dry ice and stored at $-80^{\circ} \mathrm{C}$. To tolerate individual variation, $25 \mu \mathrm{L}$ of each serum sample was collected and every 20 samples were pooled into one subgroup. Altogether, we got 5 ASD subgroups and 3 TD subgroups $(n=20)$. To avoid bias caused by gender difference, proportion of males in each subgroup was the same $(90 \%)$. The remaining serum in each sample was maintained for further individual validation.

\section{Isolation of serum L1CAM-captured extracellular vesicles (LCEVs)}

LCEVs in the pooled sera in each group were isolated as described previously [25-27] with minor modifications. Briefly, $0.5 \mathrm{ml}$ serum was incubated with $0.15 \mathrm{ml}$ of thromboplastin-D (Fisher Scientific, Inc., Hanover Park, IL) at room temperature for $60 \mathrm{~min}$. Then, $0.5 \mathrm{ml}$ calcium- and magnesium-free Dulbecco's balanced salt solution (DBS-2) with protease inhibitor cocktail (Roche Applied Sciences, Inc., Indianapolis, IN) and phosphatase inhibitor cocktail (Pierce Halt, Thermo Scientific, Inc., Rockford, IL) was added and the mixture was centrifuged at 1,500×gfor $20 \mathrm{~min}$. The supernatant was mixed with $252 \mu \mathrm{L}$ 
ExoQuick exosome precipitation solution (EXOQ; System Biosciences, Inc., Mountainview, CA), and incubated for $1 \mathrm{hr}$ at $4^{\circ} \mathrm{C}$. The resultant EVs suspension was centrifuged at $1,500 \times \mathrm{g}$ for $30 \mathrm{~min}$ at $4^{\circ} \mathrm{C}$ and the pellet was re-suspended in $150 \mu \mathrm{LBS}-2$ with the inhibitor cocktails before immunochemical enrichment of LCEVs. Each sample received $100 \mu \mathrm{L}$ 3\% BSA (1:3.33 dilution of Blocker BSA 10\% solution in DBS-2 [Thermo Scientific, Inc.]) and was incubated with $1 \mu \mathrm{g}$ mouse anti-L1CAM biotinylated antibody (clone 5G3, eBioscience, San Diego, CA) for $1 \mathrm{hr}$ at $4^{\circ} \mathrm{C}$. Following that, $25 \mu \mathrm{L}$ streptavidin-agarose resin (Thermo Scientific, Inc.) plus $50 \mu \mathrm{L} 3 \%$ BSA was added and the sample was incubated at $4^{\circ} \mathrm{C}$ for $30 \mathrm{~min}$. After centrifugation at $200 \times \mathrm{g}$ for $10 \mathrm{~min}$ at $4^{\circ} \mathrm{C}$ and removal of the supernate, $3 \% \mathrm{BSA}$ was added again, and centrifugation and supernatant removal were repeated. Each pellet was suspended in $50 \mu \mathrm{L} 0.05 \mathrm{M}$ glycine- $\mathrm{HCl}(\mathrm{pH} 3.0)$, incubated at $4^{\circ} \mathrm{C}$ for $10 \mathrm{~min}$, and re-centrifuged at $4,000 \times \mathrm{g}$ for $10 \mathrm{~min}$ at $4^{\circ} \mathrm{C}$. The obtained supernatant was transferred to a new Eppendorf tube containing $5 \mu \mathrm{L}$ of $1 \mathrm{M} \mathrm{Tris-} \mathrm{HCl}(\mathrm{pH} 8.0)$ and stored at $-80^{\circ} \mathrm{C}$.

\section{$\mathrm{nFCM}$ analysis}

LCEVs suspension at a concentration between $1 \times 10^{7} / \mathrm{ml}$ and $1 \times 10^{9} / \mathrm{ml}$ was examined using a Flow NanoAnalyzer (NanoFCM, Xiamen, China) to determine the size and quantity of particles isolated as described before (Tian et al., 2020). Briefly, the sample stream is completely illuminated within the central region of the focused laser beam, and the detection efficiency is approximately $100 \%$. The concentration of each LCEV sample was determined by employing $100 \mathrm{~nm}$ orange FluoSpheres of known particle concentration to calibrate the sample flow rate.

\section{Transmission electron microscopy (TEM)}

LCEVs solution (20-40 $\mu \mathrm{l})$ was placed on a copper mesh, post-negatively stained with $2 \%$ phosphor tungstic acid for $10 \mathrm{~min}$, and then dried for 2 min under incandescent light. The copper mesh was observed and photographed under a transmission electron microscope (H-7650 Hitachi microscope; Hitachi, Tokyo, Japan).

\section{Western blot analysis}

The LCEVs suspension was denatured in $5 \times$ sodium dodecyl sulfonate (SDS) buffer and subjected to western blot analysis (10\% SDS-polyacrylamide gel electrophoresis; $50 \mu \mathrm{g}$ protein/lane) using mouse monoclonal antibody CD63 (ab59479, Abcam, Shanghai, China), mouse monoclonal antibody CD81 (ab79559, Abcam, Shanghai, China), mouse monoclonal antibody TSG101 (ab83, Abcam, Shanghai, China) and rabbit polyclonal antibody L1CAM (13-1719-82; ThermoFisher, Shanghai, China). The proteins were visualized using the chemiluminescence detection Syngene GBox (Syngene Europe).

\section{Cytoflex Flow Cytometer analysis}

The LCEVs solution (5-10 $\mu \mathrm{l})$ was diluted by phosphate balanced solution, and diluted into three concentration gradients of 1/10, 1/100, and 1/1000. The Cytoflex Flow Cytometer (Beckman, USA) was preliminary used to detect the number of EV particles to select the most suitable dilution concentration (the average particle number is less than 10,000). Then sample was incubated with $1 \mu \mathrm{g}$ of PE-anti- 
mouse/cat CD81 (104905, Biolegend) or APC-CD63 (143905, Biolegend) antibodies for 15 minutes at room temperature. After the incubation, the Cytoflex Flow Cytometer was used to detect the expression of markers.

\section{Extraction of total RNA in LCEVs}

Total RNA in LCEVs was isolated using the Exosomal RNA isolation kit (NorgenBiotek, 58000) according to the manufacturer's instructions. Briefly, 200 $\mu$ l of the transferred supernatant containing purified LCEVs was incubated with $300 \mu \mathrm{L}$ Lysis Buffer A and $37.5 \mu \mathrm{L}$ Lysis Additive B at room temperature for $10 \mathrm{~min}$, following which $500 \mu \mathrm{L}$ of $96-100 \%$ Ethanol was added and mixed well via 10 -second vortexing. Then, $500 \mu \mathrm{L}$ of the mixture was transferred into a Mini Spin column and centrifuged at 3,000 $\times \mathrm{g}$ for $1 \mathrm{~min}$, and the remaining mixture was transferred and centrifuged by repeating the steps. After that, $600 \mu \mathrm{L}$ Wash Solution A was applied and the column was centrifuged at 3,300xg for 30 seconds twice. The spin column was then moved to a fresh $1.7 \mathrm{~mL}$ Elution tube, and $50 \mu \mathrm{L}$ Elution Solution $A$ was added. Finally, centrifugation was performed at $400 \times \mathrm{g}$ for $1 \mathrm{~min}$ and $5,800 \times \mathrm{g}$ for 2 min to obtain total RNA.

\section{Human IncRNA microarray and data analysis}

Total RNA was purified using an RNeasy Mini Kit (Qiagen, Germany) and was checked for a RIN number to inspect RNA integration with an Agilent Bioanalyzer 2100 (Agilent Technologies, Santa Clara, CA, US). LC Biotech Human IncRNA Microarray 4×180 K (Agilent Technologies; Santa Clara, CA) was utilized to detect the expression of mRNAs and IncRNAs in LCEVs. The microarray slide contained 26,083 mRNA probes and 1,05,135 IncRNA probes, and IncRNA sequencing data were obtained from Gencode, UCSC, Ensembl, Refseq, LNCIpedia, NONCODE, LNcRNA Disease, Ernas, NRED and other databases. Amplification of CRNA, fluorescent labeling and hybridization of the microarray were performed by following the protocol of Agilent Technologies. Briefly, equal amount of RNA from each subgroup was reversely transcribed into CDNA, which was then labeled with Cy3 (GE Healthcare; Biosciences, Piscataway, NJ, USA) and hybridized with the microarrayslide. The slide was then scanned with the Agilent Microarray Scanner G5761A (Agilent Technologies). Data were extracted with Feature Extraction software 12.0.3.1 (Agilent Technologies), and raw data were normalized by Quantile algorithm. Genes with a $p$ value less than 0.05 and a fold change of at least 2 were selected for further analysis. GO/KEGG pathway enrichment analyses of the target genes were performed by Fisher's exact test. The function of IncRNAs was predicted by analyzing the functional annotations of mRNAs that were highly co-expressed with IncRNAs. All raw data of our study was submitted on GEO Profiles, and GEO accession numbers is GSE186493.

\section{Small RNA library construction, sequencing and data processing}

Approximately 1 ug total RNA was used to prepare small RNA library according to the protocol of TruSeq Small RNA Sample Prep Kit (Illumina, San Diego, USA). Single-end sequencing (36 bp) was performed with an Illumina Hiseq2500 at LC-BIO (Hangzhou, China). Briefly, the raw reads were subjected to the Illumina pipeline filter (Solexa 0.3), and the dataset was further processed with an in-house program, 
ACGT101-miR (LC Sciences, Houston, Texas, USA), to remove adapter dimers, junk, low complexity, common RNA families (rRNA, tRNA, snRNA, snoRNA) and repeats. Subsequently, unique sequences with a length of 18-26 nucleotides were mapped to Homo species precursors in miRBase20.0 by BLAST search to identify known miRNAs and novel 3p-and 5p-derived miRNAs. The hairpin RNA structures containing such sequences were predicated from the flank 80 snt sequences using the RNAfold software (http://rna.tbi.univie.ac. at/cgi-bin/RNAfold.cgi). miRNA differential expression based on normalized deep-sequencing counts was analyzed using the Fisher exact test and Student $t$ test, and the significance threshold was set to be 0.01 or 0.05 . To predict the genes targeted by most abundant miRNAs, two computational target prediction algorithms (TargetScan50 and miRanda 3.3a) were used to identify miRNA binding sites. Finally, the data predicted by both algorithms were combined and the overlaps were calculated. The GO terms and KEGG pathways of these most abundant miRNAs and miRNA targets were also annotated.

\section{Quantitative real-time PCR}

All primers were designed and synthesized by Takara (Takara Biotechnology, Dalian, China). To avoid false-positive amplification of contaminated genomic DNA in the mRNA samples, all the primers spanning different exons were designed (Table 2). For mRNA, cDNA was synthesized using a PrimeScript RT reagent kit (Takara Biotechnology Co, Ltd, Dalian, China). Quantitative real-time PCR (qRT-PCR) was performed using the IQ5 real-time PCR detection system, and GADPH was taken as a control. Relative quantification of mRNA expression levels was performed using SYBR Premix Ex Taq II on an FTC3000TM System (Funglyn Biotech Inc., Toronto, Canada). For miRNA, cDNA was synthesized using the service bio RT First strand cDNA Synthesis Kit (Servicebio, Wuhan, China), qRT-PCR was carried out using the SYBR Premix Ex Taq ${ }^{\mathrm{TM}}$ II (TaKaRa), and U6 was taken as a control. PCR conditions consisted of a 5 min pre-incubation at $95^{\circ} \mathrm{C}$, followed by 40 cycles of incubation at $95^{\circ} \mathrm{C}$ for $10 \mathrm{~s}$ plus $60^{\circ} \mathrm{C}$ for $20 \mathrm{~s}$. All samples were run in triplicate and the average values were calculated. The relative levels of mRNAs, including EDNRA, SLC17A6, HTR3A, OSTC and TMEM165, as well as of miRNAs, including PC-5p139289_26 and hsa-miR-193a-5p, were calculated using the $2^{-\triangle \Delta C t}$ method. 
Table 2

All primer sequences for qRT-PCR

\begin{tabular}{|c|c|}
\hline Primer name & Sequence \\
\hline \multicolumn{2}{|l|}{ mRNA primers } \\
\hline GAPDH-F & GGAGCGAGATCCCTCCAAAAT \\
\hline GAPDH-R & GGCTGTTGTCATACTTCTCATGG \\
\hline TMEM165-F & GGCCCAGATGAAGACCTTAGC \\
\hline TMEM165-R & ACTGATATGGCAGCGACAAATG \\
\hline OSTC-F & CGTGTCCCGTTCTTAGTG \\
\hline OSTC-R & TGCCCATGTTCATCAGTC \\
\hline SLC17A6-F & GGGAGACAATCGAGCTGACG \\
\hline SLC17A6-R & TGCAGCGGATACCGAAGGA \\
\hline EDNRA- F & TCGGGTTCTATTTCTGTATGCCC \\
\hline EDNRA- R & TGTTTTTGCCACTTCTCGACG \\
\hline C12orf49-F & GCAGCCCAACAAGCAACTTC \\
\hline C12orf49-R & TGTTCTCATGCTGCACGCT \\
\hline CHRM1-F & CTCTATACCACGTACCTGCTCA \\
\hline CHRM1-R & CCGAGTCACGGAGAAGTAGC \\
\hline HTR3A-F & GAAGCCAACCACCGTATCCAT \\
\hline HTR3A-R & CCACATCCACGAACTCATTGAT \\
\hline \multicolumn{2}{|l|}{ miRNA primers } \\
\hline U6-F & GCTTCGGCAGCACATATACTAAAAT \\
\hline U6-R & CGCTTCACGAATTTGCGTGTCAT \\
\hline PC-5p-139289_26-F & ATCCAGTGCGTGTCGTG \\
\hline PC-5p-139289_26-R & TGCTGTGGCACAGGCCAG \\
\hline hsa-miR-193a-5p-F & ATCCAGTGCGTGTCGTG \\
\hline hsa-miR-193a-5p-R & TGCTTGGGTCTTTGCGGGC \\
\hline
\end{tabular}

\section{Statistics}

All statistical analyses were performed using SPSS (version 17). Group statistics are presented as mean \pm SD. The t-test for independent variables was used to examine the inter-group differences and a 
significance level of 0.05 was adopted.

\section{Community involvement}

Members of the autism community were not involved in the development of research questions, outcome measures, study design, or implementation of this trial.

\section{Results}

\section{Characterization of serum LCEVs}

Basic characteristics of the participants were shown in Table 1. Sera LCEVs in the ASD group (including 5 subgroups) and the TD group (including 3 subgroups) were isolated using L1CAM antibody mediated immunoadsorption (Figure 1). Nanoparticle tracking analysis showed a higher LCEVs concentration in the ASD group $\left(2.04 \pm 4.35 \times 10^{10} / \mathrm{ml}\right)$ than in the TD group $\left(1.20 \pm 3.28 \times 10^{10} / \mathrm{ml}\right)$. The average particle size of LCEVs was $61.50 \pm 20.71 \mathrm{~nm}$ in the ASD group and $62.07 \pm 20.75 \mathrm{~nm}$ in the TD group, showing no significant difference (Figure 2A). Under TEM, both groups of LCEVs presented a "saucer" like structure (Figure 2B). Meanwhile, LCEVs were identified by WB for EVs markers (CD63, CD81 and TSG101) (Figure 2C) as well as L1CAM (Figure 2D). In addition, Cytoflex Flow Cytometer analysis revealed that LCEVs marked with CD63 and CD81 accounted for $57.78 \%$ and $76.63 \%$ of the total LCEVs in TD, and $55.82 \%$ and $69.29 \%$ of the total LCEVs in ASD (Supplementary figure 1).

\section{Differential expression and bioinformatic analysis of mRNAs in ASD serum LCEVs}

Based on IncRNA microarray detection and original data normalization, air-wise Pearson's correlation coefficients of all RNAs among subgroups were shown in Figure 3A. The coefficients between the biological replicates (subgroups) within each group were obviously higher than those between the two groups (Figure 3A). mRNAs and IncRNAs with at least twofold differential expression and a P value of less than 0.05 were subjected to further examination. This resulted in 167 up-regulated and 1251 downregulated mRNAs in ASD sera LCEVs (Figure 3B and Supplementary Table 1). Hierarchical clustering analysis (HCA) of these 1418 differentially expressed mRNAs (DEmRs) showed similar expression profiles among the biological replicates within each group but differential profiles between the two groups (Figure 3C). Principal component analysis (PCA) of the DEmRs showed that the two groups were separated and the biological replicates in the TD group clustered more closely than those in the ASD group (Figure 3D). To characterize the distribution of genes for DEmRs on chromosomes and to reveal the susceptible chromosomes, the genes on each chromosome were counted and the ratio of the number of such genes to the total number of genes present on the chromosome (data from Human Genome Resources at NCBI, GRCh37) was calculated. As a result, chromosomes 1 and 2 had the largest number of genes for DEmRs, while chromosomes 21 and $Y$ had the least number of such genes. However, genes with the maximum ratio were on chromosomes 17 and 5, and those with the minimum ratio were on 
chromosomes 13 and $\mathrm{Y}$ (Figure 3E). GO annotation of DEmRs showed that their products were mainly distributed in cytoplasm, nucleus and plasma membrane; were able to bind with proteins, metal ions and DNA; and were involved mainly in DNA-dependent transcription, small molecule metabolism, transcriptional regulation and other biological processes (Figure 3F). KEGG analysis revealed that DEmRs participated in mainly three types of processes: (1) the signal transduction processes, such as MAPK signaling pathway, calcium signaling pathway, PI3K-Akt signaling pathway and cAMP signaling pathway; (2)neuron-related pathways such as neuroactive reception-ligand interaction, axon guidance and synaptic vesicle circulation; and (3) glycosylation related pathways such as $\mathrm{N}$-glycan biosynthesis, endoplasmic reticulum protein processing, sugar binding and glycosaminoglycan degradation (Figure 3G).

\section{Differential expression and functional prediction of IncRNAs in ASD serum LCEVs}

According to the results of IncRNA microarray, 239 IncRNAs were significantly up-regulated and 1506 IncRNAs were significantly down-regulated in ASD serum LCEVs (Figure 4A and Supplementary Table 2). HCA of these 1745 differentially expressed IncRNAs (DElnRs) showed similar expression profiles among the biological replicates within each group but differential profiles between the two groups (Figure 4B). PCA of the DElnRs separated the subgroups into TD and ASD groups as their natural grouping (Figure 4C). To characterize the distribution of DElnRs on chromosomes and to reveal the susceptible chromosomes, the DElnRs on each chromosome were counted and the ratio of the number of DElnRs to the length of that chromosome was calculated (data from Human Genome Resources at NCBI, GRCh37; the unit of length is $\mathrm{Mbp}$ ). It was found that chromosomes 1 and 2 had the largest number of DElnRs and chromosomes 21 and $Y$ had the least number of DElnRs; whereas, chromosomes 19 and 17 had the maximum ratio and chromosomes $X$ and $Y$ had the minimum ratio (Figure 4D). Genes with a distance of less than $100 \mathrm{~kb}$ from IncRNA were regarded as the target genes for cis-acting. As a result, $382 \mathrm{DElnRs}$ were predicted to be positively or negatively correlated with their target genes $(R>0.8)$ (Supplementary Table 3). Of these genes, 107 were also DEmRs (Figure 4E and Supplementary Table 3). Double-omic analysis (https://www.omicstudio.cn/tool) of the 107 pairs of DElnR-DEmR revealed that $81.3 \%$ pairs were positively correlated and commonly down-regulated in ASD (Figure 4F). GO annotation showed that these 107 target genes were mainly cytoskeleton-related proteins (Figure $4 \mathrm{G}$ ) performing functions such as auxiliary transport protein activity, protein binding and translation regulation (Figure $4 \mathrm{H}$ ), and were involved in protein metabolism, transport, and cell growth and/or maintenance (Figure 4I).

\section{Neuron-related network and significant markers in ASD}

Notably but not unexpectedly, 104 DEmRs were related to neural molecular pathways, of which 19 were up-regulated (e.g., EDNRA) and 85 (e.g., SLC17A6 AND HTR3A) were down-regulated (Figure 5A). For IncRNAs, the expression levels of SNHG1, Inc-C20orf201-1 and Inc-TRPV5-1 were positively correlated with their target genes, namely CHRM1, OPRL1 and EPHB6 (Figure 5B). According to GO and KEGG analyses, these neuron-related RNAs were mainly involved in neuroactive ligand-receptor interaction 
(e.g.,ADORA2A and EDNRA), pathways of neurodegeneration (e.g., CHRM1and SNHG1 [IncRNA]), glutamatergic synapse (e.g., GRIA4 and HOMER2), axon guidance (e.g.,EPHB6 and Inc-TRPV5-1 [IncRNA]), synaptic vesicle cycle (e.g., ATP6V1G1 and SLC17A6), dendrite formation (e.g., TENM2 and IGSF9B), neuron projection development (e.g., OPRL1 and Inc-C20orf201-1 [IncRNA]), neuron migration (e.g., BARHL1 and DISC1), neuron apoptotic process (e.g.,CLN3 and BCL2L11), calcium signaling pathway (e.g., ADORA2A and CCKAR), as well as MAPK signaling pathway (e.g., ERBB3 and TNFRSF1A). All of these processes formed a neuron-related signaling network implicated in the molecular mechanisms of ASD (Figure 5C). The most enriched pathway was neuroactive ligand-receptor interaction, involving 5 up-regulated mRNAs (e.g., EDNRA) and 19 down-regulated mRNAs (e.g., HTR3A) in ASD (Figure 5D). To validate the differential expression of these RNAs, EDNRA, SLC17A6 and HTR3A were randomly selected and examined by qRT-PCR in individual serum samples. As a result, the level of EDNRA was significantly higher in ASD than in TD $(p=0.001)$, while those of SLC17A6 $(p=0.03)$ and HTR3A $(p=0.007)$ were significantly lower (Figure $5 \mathrm{E})$. These were consistent with the results of IncRNA microarray.

\section{Glycan-related network and candidate markers in ASD}

Most eukaryotic proteins are modified by covalent addition of glycan molecules, which modulates the structures and functions of the proteins. Glycans are synthesized in the presence of glycosyltransferases, sulfonyltransferases, glycosidases and other glycan-binding proteins; aberrant expression of these enzymes may result in many complicated pathological conditions, such as inflammation, diabetes, cancer and neurological abnormality(Kronimus, Dodel, Galuska, \& Neumann, 2019; Pinho \& Reis, 2015). According to IncRNA microarray and bioinformatic analysis, 54 DEmRs were carbohydrate-related genes, of which 5 (e.g., HPSE, and GALM) were significantly up-regulated and others (e.g., OSTC and MAN1B1) were significantly down-regulated in ASD (Figure 6A). DElnRs including LOC101927919, Inc-KIAA1919-1, Inc-SHCBP1L-1 and RP11-177J6.1 were positively correlated with their predicted target genes NUS1, KIAA1919, NPL and TMEM165, respectively (Figure 6B). Based on GO and KEGG analysis, these DEmRs and DElnRs were mainly involved in carbohydrate metabolic process (e.g., HPSE and SLC37A4), protein N-linked glycosylation (e.g., MGAT5 and OSTC), carbohydrate binding (e.g., MSIGLEC5 and CLEC1A), glycolysis (e.g., GALM and PGM1), glycosaminoglycan metabolic process (e.g., CHST12 and HPSE), and glycolipid metabolic process (e.g., NEU1 and ST8SIA6) (Figure 6C). Expressions of OSTC, MAN1B1 and MGAT5 were down-regulated in N-glycan biosynthesis implicated in ASD (Figure 6D). To validate the differential expression of these RNAs, OSTC and TMEM165 of LCEVs were randomly selected and examined by qRT-PCR in individual serum samples. The results showed that the levels of OSTC and TMEM165were significantly lower in the ASD children ( $p=0.001$ and $p=0.02)$ (Figure 6E).

\section{Expression of miRNA in serum LCEVs}

A total of 4310 mature microRNAs (miRNAs) were examined in the serum LCEVs, among which 150 were present in all subgroups (Supplementary Table 4). The miRNA with the highest concentration across all the subgroups was hsa-miR-21-5p_R+1, accounting for $6.9 \%$ in TD and $8.5 \%$ in ASD in terms of the 
normalized read counts (Figure 7A). Unpaired two-tailed Student's t test identified 10 miRNA sequences with significant differences $(p<0.05)$ between ASD and TD (Fig. 7B). The sequence

GATTTCTTCCCAGTGCTCTGA was aligned to two pre-miRNAs and was given two names: mmu-mir-6240p3_1ss8GT and mmu-mir-6240-p5_1ss8GT. Of these 11 miRNA, the one with the biggest variation was PC-3p-38497_124, which was remarkably up-regulated (fold change $=20.32, p=0.029$ ) in ASD relative to TD (Figure 7B).The miRNA with the most significant change was PC-5p-139289_26, which was absent in the TD subgroups ( $p=0.0056$ )(Fig. 7B). Two other miRNAs (PC-3p-275123_15 and PC-5p-149427_24) were up-regulated in ASD, and another seven miRNAs (e.g., hsa-miR-193a-5p and mmu-mir-6240-p3_1ss8GT) were relatively down-regulated (Figure 7B). HCA of these differentially expressed miRNAs (DEmiRs) revealed similar expression profiles among the biological replicates within each group but differential profiles between the ASD and TD groups (Figure 7C). PCA showed that the biological replicates in the TD group clustered more closely (Figure 7D).

Targets of these 11 DEmiRs were predicted by both TargetScan 50 and miRanda 3.3a algorithms. According to the results of IncRNA microarray, 25 of the predicted targets were DEmRs and 45 were DElnRs (Supplemental Figure 2 and Supplementary Table 5). Notably, $86.2 \%$ of the DEmRs and $71.1 \%$ of the DElnRs were down-regulated in serum LCEVs of ASD. Two miRNAs, namely PC-5p-139289_26 and hsa-miR-193a-5p, had the largest number of predicted targets with high confidence (TargetScanscore>90). For hsa-miR-193a-5p, 3 mRNAs (PIGZ, CEBPG and NETO2) were down-regulated and 2 (PAQR3 and OMD) were up-regulated, while 14 IncRNAs (e.g., CTC-340A15.2 and Inc-CLDN20-3) were down-regulated and 3 (ENST00000532430, CAND1.11 and EPS15L1) were up-regulated in the serum LCEVs of ASD (Figure 7E). For PC-5p-139289_26, 11 mRNAs (e.g., ING3 and BRD7) were downregulated and 2 (EDNRA and SCUBE3) were up-regulated, while 11 IncRNAs (e.g., RP11-401P9.1 and LOC400043) were down-regulated and 4 (e.g., RSU1P2 and LOC101927027) were up-regulated in ASD (Figure 7E). GO annotation revealed that most targets were related to signal transduction (41\%) and cell communication (36\%). The most enriched annotation was glutathione synthesis and recycling (fold enrichment=263). Other enriched annotations included mannosyltransferase activity, attachment of GPI anchor to uPAR, gamma-glutamyl cycle, prostanoid ligand receptors and eicosanoid ligand-binding receptors (fold enrichment>80) (Figure 7F). To validate the differential expression of these DEmiRs, PC5p-139289_26 and hsa-miR-193a-5p were randomly selected and examined by qRT-PCR in individual serum samples. The results showed that the expression of PC-5p-139289_26 increased significantly $(p=0.0008)$ and that of hsa-miR-193a-5p decreased significantly $(p=0.003)$ in ASD (Figure 7G). These results were consistent with those of the RNA sequencing.

\section{Discussion}

At present, the diagnosis of ASD is still based on symptom evaluation, as the underlying pathological mechanism remains unclear. There are no blood-based diagnostic tools or approved drugs for ASD. Research to identify reliable biological markers of disease status and symptomology is therefore urgently needed. Neurobiological systems critical to social functioning are arguably the most promising biological sources for ASD biomarkers and therapeutic targets. However, existing methods for brain detection 
mostly relied on autopsy or animal models, which are limited because of poor timeliness and species differences. Most cells in the nervous system, including neurons, astrocytes, oligodendrocytes and microglia, secrete EVs under normal or pathological conditions. EVs can reflect the host cell proteins and nucleic acids at the time of secretion and can diffuse across the blood brain barrier into the periphery. Serum/plasma LCEVs can be captured by antibodies directed against the cell surface protein L1CAM embedded in the vesicle membrane(Goetzl et al., 2018; Goetzl et al., 2020; Nogueras-Ortiz et al., 2020). Although investigation of LCEVs is relatively novel, attractive evidence from other fields suggests that such investigation can afford insight into the pathological mechanisms and processes associated with Alzheimer's disease and depressive disorder(Kuwano et al., 2018; Song et al., 2020). A recent study developed a panel of single-molecule array assays to evaluate the use of L1CAM for neuron-derived EV (LCEVs) isolation, and demonstrated that L1CAM behaved as a soluble protein, not as an EV-associated protein, and therefore recommend against its use as a marker in LCEV isolation protocols(Norman et al., 2021). It is mentionable that they fractionated plasma and cerebrospinal fluid using size exclusion chromatography (SEC) and density gradient centrifugation (DGC), and found that L1CAM expression overlapped at the tails of earlier fractions with the later fractions. Actually, in this study, total EVs were extracted from serum preferentially and then were used to isolate LCEVs by immunoprecipitation to avoid soluble protein interference as much as possible. The average particle size of LCEVs was $61.50 \pm 20.71$ $\mathrm{nm}$ in the ASD group and $62.07 \pm 20.75 \mathrm{~nm}$ in the TD group, which was consistent with a latest study that reported that LCEVs were smaller than other EVs isolated from plasma $(p<0.0001)$ (Saeedi et al., 2021). Overall, LCEVs can be enriched by L1CAM antibody in peripheral blood but the size was smaller than most EVs without L1CAM.

Thus far, a putative speech and language region at chromosome 7q31-q33 seems most strongly linked to autism. Cytogenetic abnormalities at the 15q11-q13 locus are fairly frequent in people with autism, and a "chromosome 15 phenotype" is described in individuals with chromosome 15 duplications(Nakatani et al., 2009). Some candidate genes are considered located at chromosomes 7q22-q33 and 15q11q13(Muhle, Trentacoste, \& Rapin, 2004), and 21 genes in chromosomal 8p region are identified as most likely to contribute to neuropsychiatric disorders and neurodegenerative disorders(Tabares-Seisdedos \& Rubenstein, 2009). Variant alleles of the serotonin transporter gene (5-HTT) on chromosome 17q11-q12 are more frequent in individuals with autism than in healthy people(Nakatani et al., 2009). In addition, many mutations on NLGN4X, an X-linked cell adhesion molecule, result in ASD (Nguyen et al., 2020). In the present study, chromosome 17 was the commonly and mostly enriched chromosome for both DEmRs and DElnRs in ASD. A large portion of the DEmRs on chromosome 17 participates in cell communication and signal transduction, which are essential for synapse formation and neurotransmitter release. Abnormal expression of such mRNAs implies the abnormality of these functions in ASD.

Brain-derived EVs carry and release multiple molecules related to neuronal function and neurotransmission in the brain, which is beneficial for the reciprocal communication between neural cells (e.g., neuron-glia interactions), synaptic plasticity, neuronal development, and neuroimmune communication. In the present study, 104 DEmRs were annotated to be related to neuroactive ligandreceptor interaction, pathways of neurodegeneration, glutamatergic synapse, axon guidance, synaptic 
vesicle cycle, dendrite, neuron projection development, neuron migration and apoptotic process. Most $(81.7 \%)$ of these neuron-related mRNAs were down-regulated in ASD. As demonstrated in the pathway of neuroactive ligand-receptor interaction (Fig. 3H), 5 receptors (e.g., EDNRA) were up-regulated and 19 (e.g., HTR3A) were down-regulated in ASD. A previous study reported that neuropeptide receptor gene expression was lower in children with autism and the lower neuropeptide receptor gene expression predicted greater social impairments and greater stereotyped behaviors(Oztan et al., 2018). We found that 5-hydroxytryptamine receptor 3A (HTR3A) significantly decreased in the ASD serum LCEVs in this study. HTR3A is one of the receptors for 5-hydroxytryptamine (serotonin), a biogenic hormone that also functions as a neurotransmitter and a mitogen. Ample evidence suggests that levels of serotonin and serotonin transporter (SERT) increase significantly in autistic children than in gender and age-matched non-autistic children (Abdulamir, Abdul-Rasheed, \& Abdulghani, 2018; Meyyazhagan et al., 2020). It thus can be hypothesized that increase of serotonin and SERT may be a kind of cell self-help that compensates for the loss of receptors, but it needs to be experimentally confirmed in the future. Another specific signature is the decreased expression of vesicular glutamate transporter 2 (SLC17A6) in the ASD serum LCEVs. Receptors for glutamate (Glu), GRIK5, GRIK2 and GRIA4 were also down-regulated. Glu acts as an excitatory neurotransmitter at many synapses in the central nervous system. SLC17A6 mediates the uptake of Glu into synaptic vesicles at presynaptic nerve terminals of excitatory neural cells. The postsynaptic actions of Glu are mediated by a variety of receptors expressed on postsynaptic cell membrane. Emerging evidence suggests that imbalance between excitatory (Glu-mediated) and inhibitory (GABA-mediated) neurotransmission may be a common pathophysiological mechanism in ASD(Horder et al., 2018; Rojas, 2014). These studies, together with the findings in the present study, suggest that reduction in the expression of Glu transporter and receptors might be the main reason for the abnormalities of Glu-mediated neurotransmission and hence a therapeutic target in ASD.

Glycans and their conjugates (glycoproteins, proteoglycans and glycolipids) are major constituents of the neural cell membrane and extracellular matrix (ECM). Glycans and glycoconjugates participate in nearly every biological process in the developing brain. A potential link between ASD and changes in glycosylation was first observed in patients with congenital glycosylation disorders (CDGs)(Freeze, Eklund, Ng, \& Patterson, 2015). Recent advances in genome sequencing have identified many genetic variants that occur in genes encoding glycosylated proteins (proteoglycans or glycoproteins) or enzymes involved in glycosylation (glycosyltransferases and sulfotransferases)(Dwyer \& Esko, 2016; Yu et al., 2013). However, it remains unknown whether "glycogene" variants cause changes in glycosylation and whether they contribute to the etiology and pathogenesis of ASDs. In the present study, we analyzed the whole transcriptome of serum LCEVs in ASD to screen potential biomarkers and explore the important molecular events in brain neurons of ASD children. Our results showed that a total of 54 DEmRs (3.8\%) were glycogenes, and most of them (90.7\%) were down-regulated in ASD. The 54 DEmRs mainly participated in carbohydrate metabolic process, protein $\mathrm{N}$-linked glycosylation, carbohydrate binding, glycolysis, glycosaminoglycan metabolic process and glycolipid metabolic process. Thereinto, OSTC, MAN1B1 and MGAT5, translating to key enzymes for N-linked glycosylation, were significantly downregulated in ASD. In our previous study, we found a significant decrease of STL binding glycans or 
glycoproteins that contain trimers and tetramers of GlcNAc (core structure of $\mathrm{N}$-glycans) in ASD versus in TD (fold change $=0.54, p=0.0057$ ) (Qin et al., 2017). In all, no matter at the gene level, the transcription level, or the level of translation and post-translation modification, abnormalities of glycosylation and carbohydrate metabolism might be an important molecular mechanism of ASD. Moreover, the decrease of receptors and transporters of neurotransmitters may be related with the decrease of glycogenes as most of the receptors and transporters are highly glycosylated. OSTC is a subunit of the oligosaccharyl transferase (OST) complex that catalyzes the initial transfer of a defined glycan (Glc3Man9GIcNAc2 in eukaryotes) from the lipid carrier dolichol-pyrophosphate to an asparagine residue within an Asn-XSer/Thr consensus motif in nascent polypeptide chains. In the present study, expression of OSTC significantly decreased in ASD serum LCEVs, suggesting it as a candidate biomarker for ASD diagnosis.

Recent studies have shown that abnormal expression of miRNAs could be involved in the underlying pathogenesis of ASD. miRNAs are small noncoding mRNAs that regulate gene expression and are often linked to biological processes and implicated in neurodevelopment. A dozen of miRNAs, such as miRNA$125 \mathrm{~b}$ and miRNA-132, have been observed to regulate the expression of ASD risk genes, act differently on the morphology of the spine and synaptic plasticity in brain neurons, and participate in ASD etiopathogenesis(Schepici, Cavalli, Bramanti, \& Mazzon, 2019). However, compared with mRNA and IncRNA, fewer miRNAs were found differentially expressed in ASD serum LCEVs in the present study. Among 11 DEmiRs, PC-5p-139289_26 was significantly up-regulated and hsa-miR-193a-5p was significantly down-regulated in ASD, and both of them had the largest number of predicted targets that were differentially expressed in ASD, indicating that these two miRNAs might play important roles in ASD. These targets were mostly involved in glutathione synthesis and recycling and mannosyltransferase activity, which are closely correlated with synthesis of Glu and glycans involved in the neuron- and glycan-related networks in ASD. However, the relationships between miRNAs and their target genes have not yet been verified.

This study might have some limitations that merit consideration. Firstly, we did not examine the correlation between the expression of candidate biomarkers and disease severity. This would be addressed in our future research. Secondly, we collected only one blood sample per participant (due to the invasive nature of venipuncture, particularly in children), which limited our ability to assess withinindividual consistency of our biological measures. Thirdly, some of our participants were not medicationfree. Even though their medications were stable (for at least four weeks) before blood collection, it is possible that our results might be influenced by the medication status. Fourthly, our samples were mainly from a single hospital. Although it is one of the few famous hospitals in the northwest of China that treat ASD children from five neighboring provinces, most of its patients are still from the local regions. Further research involving participants from multiple areas would be a great addition to the present study.

\section{Conclusions}

In short, 1418 mRNAs, 1745 IncRNAs and 11 miRNAs were identified differentially expressed in the serum LCEVs from the ASD children versus from the TD children. Most of these RNAs were down-regulated and 
involved in neuron-related and glycan-related networks implicated in ASD. Levels of potential markers, including EDNRA, SLC17A6, HTR3A, OSTC, TMEM165, C12orf49, PC-5p-139289_26, and hsa-miR-193a$5 p$, changed significantly in the ASD children. Whole-transcriptome analysis of serum LCEVs reveals neural and glycosylation changes in ASD, which could serve as predictive biomarkers and provide information for understanding the molecular mechanisms of ASD. Hopefully, our results may provide reference for future diagnostic and therapeutic management of the disease.

\section{List Of Abbreviations}

ASD: Autism Spectrum Disorder

EVsロExtracellular vesicles

L1CAM: Neural cell adhesion molecule L1

LCEVs: L1CAM-captured extracellular vesicles

TD: Typically developed

DSM: Diagnostic and Statistical Manual of Mental Disorders

APOD: Apolipoprotein D

DBS-2: Dulbecco's balanced salt solution

SDS: Sodium Dodecyl Sulfonate

qRT-PCR: Quantitative real-time PCR

HCA: Hierarchical clustering analysis

PCA: Principal component analysis

DEmRs: Differentially expressed mRNAs

DElnRs: Differentially expressed IncRNAs

DEmiRs: Differentially expressed miRNAs

HTR3A: 5-hydroxytryptamine receptor 3A

SERT: Serotonin transporter

SLC17A6: Vesicular glutamate transporter 2

Glu: Glutamate 
ECM: Extracellular matrix

CDGs: Congenital glycosylation disorders

OST: Oligosaccharyl transferase

\section{Declarations}

\section{Acknowledgements}

The authors sincerely thank all the participants.

\section{Funding}

This work was supported by National Natural Science Foundation of China(No. 81401137 and No. 81972603), and the Natural Science Foundation of Shaanxi Province (No.2017JM8112).

\section{Availability of data and materials}

The datasets used and/or analyzed during the current study are available from the corresponding author on reasonable request.

\section{Code Availability}

Not applicable.

\section{Author information}

\section{Affiliations}

Department of cell Biology and Genetics, School of Basic Medical Sciences, Xi'an Jiaotong University, Shaanxi, Xi'an 71 Department 0061, P. R. China

Yannan Qin, Li CaoهShuang Cai, Jinyuan Zhang, Bo Guo, Fei Wu, Lingyu Zhao, Wen Li, Lei Ni \& Chen Huang

Biomedical Experimental Center of Xi'an Jiaotong University, Shaanxi, China

Liying Liu, Xiaofei Wang \& Chen Huang

Xi'an Children's Hospital, Xi'an Jiaotong University, Shaanxi, China

Haiqing Zhang \& Yanni Chen

\section{Authors' contributions}


Y.Q. carried out analysis of data from IncRNA microarray and RNA sequencing, generated the graphs, and wrote the manuscript; L.C. took charge of preparation of serum samples, isolation and characterization of serum LCEVs, and qRT-PCR; H.Z. collected samples from patients and summarize the clinical information of patients; S.C., J.Z. and L.Z. participated in data analysis; B.G. performed extraction of LCEVs; F.W., W.L. and X.W. participated in bioinformatics analysis; L.N. and L.L. modified the draft of this paper; Y.C. performed ASD diagnosis and sample collection; and C.H. participated in the design of the project, coordination of the staff and revision of the manuscript. All authors read and approved the final manuscript.

\section{Ethics declarations}

\section{Ethics approval and consent to participate}

The Ethics Committee of Xi'an Jiaotong University (Xi'an, China) provided institutional ethical approval for this study, and the number is 20190037 . Here, we provide file of permission.

\section{Competing interests}

The authors declare that they have no competing interests.

\section{Consent for Publication}

All parents of the participants signed written informed consent.

\section{Data availability}

The data supporting the fingdings of this study can be obtained from the corresponding author upon reasonable request.

\section{Publisher's Note}

Springer Nature remains neutral with regard to jurisdictional claims in published maps and institutional affiliations.

\section{References}

1. Abdulamir HA, Abdul-Rasheed OF, Abdulghani EA (2018) Serotonin and serotonin transporter levels in autistic children. Saudi Med J 39(5):487-494. doi:10.15537/smj.2018.5.21751

2. Alessio N, Brigida AL, Peluso G, Antonucci N, Galderisi U, Siniscalco D (2020) Stem Cell-Derived Exosomes in Autism Spectrum Disorder. Int J Environ Res Public Health 17(3). doi:10.3390/ijerph17030944 
3. Andjus P, Kosanovic M, Milicevic K, Gautam M, Vainio SJ, Jagecic D, Zavan B (2020) Extracellular Vesicles as Innovative Tool for Diagnosis, Regeneration and Protection against Neurological Damage. International Journal of Molecular Sciences, 21(18). doi:ARTN 685910.3390/ijms21186859

4. American Psychiatric Association (2013) Diagnostic and statistical manual of mental disorders (5th ed.). https://doi.org/10.1176/appi.books.9780890425596

5. Dwyer CA, Esko JD (2016) Glycan susceptibility factors in autism spectrum disorders. Mol Aspects Med 51:104-114. doi:10.1016/j.mam.2016.07.001

6. Fayazi N, Sheykhhasan M, AsI SS, Najafi R (2021) Stem Cell-Derived Exosomes: a New Strategy of Neurodegenerative Disease Treatment. Mol Neurobiol 58(7):3494-3514. doi:10.1007/s12035-02102324-x

7. Filippone A, Pratico D (2021) Endosome Dysregulation in Down Syndrome: A Potential Contributor to Alzheimer Disease Pathology. Ann Neurol 90(1):4-14. doi:10.1002/ana.26042

8. Freeze HH, Eklund EA, Ng BG, Patterson MC (2015) Neurological Aspects of Human Glycosylation Disorders. Annu Rev Neurosci 38:105-. doi:10.1146/annurev-neuro-071714-034019

9. Fries GR, Quevedo J (2018) Exosomal MicroRNAs as Potential Biomarkers in Neuropsychiatric Disorders. Methods Mol Biol 1733:79-85. doi:10.1007/978-1-4939-7601-0_6

10. Goetzl EJ, Abner EL, Jicha GA, Kapogiannis D, Schwartz JB (2018) Declining levels of functionally specialized synaptic proteins in plasma neuronal exosomes with progression of Alzheimer's disease. FASEB J 32(2):888-893. doi:10.1096/fj.201700731R

11. Goetzl EJ, Peltz CB, Mustapic M, Kapogiannis D, Yaffe K (2020) Neuron-Derived Plasma Exosome Proteins after Remote Traumatic Brain Injury. J Neurotrauma 37(2):382-388. doi:10.1089/neu.2019.6711

12. Hill AF (2019) Extracellular Vesicles and Neurodegenerative Diseases. J Neurosci 39(47):9269-9273. doi:10.1523/JNEUROSCI.0147-18.2019

13. Horder J, Petrinovic MM, Mendez MA, Bruns A, Takumi T, Spooren W, Murphy DG (2018) Glutamate and GABA in autism spectrum disorder-a translational magnetic resonance spectroscopy study in man and rodent models. Translational Psychiatry, 8. doi:ARTN 10610.1038/s41398-018-0155-1

14. Hyman SL, Levy SE, Myers SM, Kuo DZ, Apkon S, Davidson LF, Pediat SDB (2020) Identification, Evaluation, and Management of Children With Autism Spectrum Disorder. Pediatrics, 145(1). doi:ARTN e20193447 1542/peds.2019-3447

15. Jin X, Simmons SK, Guo A, Shetty AS, Ko M, Nguyen L, Arlotta P (2020) In vivo Perturb-Seq reveals neuronal and glial abnormalities associated with autism risk genes. Science, 370(6520), 1057-+. doi:ARTN eaaz606310.1126/science.aaz60603

16. Kramer J, Beer M, Bode H, Winter B (2020) Two Novel Compound Heterozygous Mutations in the TRAPPC9 Gene Reveal a Connection of Non-syndromic Intellectual Disability and Autism Spectrum Disorder. Front Genet 11:972. doi:10.3389/fgene.2020.00972

17. Kronimus Y, Dodel R, Galuska SP, Neumann S (2019) IgG Fc N-glycosylation: Alterations in neurologic diseases and potential therapeutic target? J Autoimmun 96:14-23. doi:10.1016/j.jaut.2018.10.006 
18. Kuwano N, Kato TA, Mitsuhashi M, Sato-Kasai M, Shimokawa N, Hayakawa K, Kanba S (2018) Neuron-related blood inflammatory markers as an objective evaluation tool for major depressive disorder: An exploratory pilot case-control study. J Affect Disord 240:88-98. doi:10.1016/j.jad.2018.07.040

19. Lin KZ, Liu H, Roeder K (2021) Covariance-based sample selection for heterogeneous data: Applications to gene expression and autism risk gene detection. J Am Stat Assoc 116(533):54-67. doi:10.1080/01621459.2020.1738234

20. Lord C, Elsabbagh M, Baird G, Veenstra-Vanderweele J (2018) Autism spectrum disorder. Lancet (London, England) 392(10146):508-520. doi:10.1016/S0140-6736(18)31129-2

21. Meyyazhagan A, Balasubramanian B, Easwaran M, Alagamuthu KK, Shanmugam S, Bhotla HK, Cacabelos R (2020) Biomarker study of the biological parameter and neurotransmitter levels in autistics. Mol Cell Biochem 474(1-2):277-284. doi:10.1007/s11010-020-03851-2

22. Muhle R, Trentacoste SV, Rapin I (2004) The genetics of autism. Pediatrics, 113(5), e472-e486. Retrieved from https://pubmed.ncbi.nlm.nih.gov/15121991

23. Nakatani J, Tamada K, Hatanaka F, Ise S, Ohta H, Inoue K, Takumi T (2009) Abnormal Behavior in a Chromosome-Engineered Mouse Model for Human 15q11-13 Duplication Seen in Autism. Cell 137(7):1235-1246. doi:10.1016/j.cell.2009.04.024

24. Nguyen TA, Wu KW, Pandey S, Lehr AW, Li Y, Bemben MA, Roche KW (2020) A Cluster of AutismAssociated Variants on X-Linked NLGN4X Functionally Resemble NLGN4Y. Neuron 106(5):759-. doi:10.1016/j.neuron.2020.03.008

25. Nogueras-Ortiz CJ, Mahairaki V, Delgado-Peraza F, Das D, Avgerinos K, Eren E, Kapogiannis D (2020) Astrocyte- and Neuron-Derived Extracellular Vesicles from Alzheimer's Disease Patients Effect Complement-Mediated Neurotoxicity. Cells 9(7). doi:10.3390/cells9071618

26. Norman M, Ter-Ovanesyan D, Trieu W, Lazarovits R, Kowal EJK, Lee JH, Walt DR (2021) L1CAM is not associated with extracellular vesicles in human cerebrospinal fluid or plasma. Nat Methods 18(6):631-634. doi:10.1038/s41592-021-01174-8

27. Oztan O, Jackson LP, Libove RA, Sumiyoshi RD, Phillips JM, Garner JP, Parker KJ (2018) Biomarker discovery for disease status and symptom severity in children with autism.

Psychoneuroendocrinology 89:39-45. doi:10.1016/j.psyneuen.2017.12.022

28. Perets N, Oron O, Herman S, Elliott E, Offen D (2020) Exosomes derived from mesenchymal stem cells improved core symptoms of genetically modified mouse model of autism Shank3B. Molecular autism 11(1):65. doi:10.1186/s13229-020-00366-x

29. Pinho SS, Reis CA (2015) Glycosylation in cancer: mechanisms and clinical implications. Nat Rev Cancer 15(9):540-555. doi:10.1038/nrc3982

30. Qin Y, Chen Y, Yang J, Wu F, Zhao L, Yang F, Huang C (2017) Serum glycopattern and Maackia amurensis lectin-II binding glycoproteins in autism spectrum disorder. Sci Rep 7:46041.

doi:10.1038/srep46041

Page 22/33 
31. Rojas DC (2014) The role of glutamate and its receptors in autism and the use of glutamate receptor antagonists in treatment. J Neural Transm 121(8):891-905. doi:10.1007/s00702-014-1216-0

32. Saeedi S, Nagy C, Ibrahim P, Theroux JF, Wakid M, Fiori LM, Turecki G (2021) Neuron-derived extracellular vesicles enriched from plasma show altered size and miRNA cargo as a function of antidepressant drug response. Mol Psychiatry. doi:10.1038/s41380-021-01255-2

33. Saint-Pol J, Gosselet F, Duban-Deweer S, Pottiez G, Karamanos Y (2020) Targeting and Crossing the Blood-Brain Barrier with Extracellular Vesicles. Cells 9(4). doi:10.3390/cells9040851

34. Sanders SJ, Murtha MT, Gupta AR, Murdoch JD, Raubeson MJ, Willsey AJ, State MW (2012) De novo mutations revealed by whole-exome sequencing are strongly associated with autism. Nature 485(7397):237-241. doi:10.1038/nature10945

35. Schepici G, Cavalli E, Bramanti P, Mazzon E (2019) Autism Spectrum Disorder and miRNA: An Overview of Experimental Models.Brain Sciences, 9(10). doi:ARTN 265 3390/brainsci9100265

36. Song ZQ, Xu YF, Deng W, Zhang L, Zhu H, Yu P, Qin C (2020) Brain Derived Exosomes Are a DoubleEdged Sword in Alzheimer's Disease. Frontiers in Molecular Neuroscience, 13. doi:ARTN 7910.3389/fnmol.2020.00079

37. Sun X, Allison C, Wei LP, Matthews FE, Auyeung B, Wu YY, Brayne C (2019) Autism prevalence in China is comparable to Western prevalence. Molecular autism, 10. doi:ARTN 710.1186/s13229-0180246-0

38. Tabares-Seisdedos R, Rubenstein JLR (2009) Chromosome $8 p$ as a potential hub for developmental neuropsychiatric disorders: implications for schizophrenia, autism and cancer. Mol Psychiatry 14(6):563-589. doi:10.1038/mp.2009.2

39. Tian Y, Gong MF, Hu YY, Liu HS, Zhang WQ, Zhang MM, Yan XM (2020) Quality and efficiency assessment of six extracellular vesicle isolation methods by nano-flow cytometry. Journal of Extracellular Vesicles, 9(1). doi:Artn 1697028 1080/20013078.2019.1697028

40. Tsilioni I, Theoharides TC (2018) Extracellular vesicles are increased in the serum of children with autism spectrum disorder, contain mitochondrial DNA, and stimulate human microglia to secrete IL1beta. J Neuroinflammation 15(1):239. doi:10.1186/s12974-018-1275-5

41. Velmeshev D, Schirmer L, Jung D, Haeussler M, Perez Y, Mayer S, Kriegstein AR (2019) Single-cell genomics identifies cell type-specific molecular changes in autism. Science 364(6441):685- . doi:10.1126/science.aav8130

42. Vorstman JAS, Parr JR, Moreno-De-Luca D, Anney RJL, Nurnberger JI Jr, Hallmayer JF (2017) Autism genetics: opportunities and challenges for clinical translation. Nat Rev Genet 18(6):362-376. doi:10.1038/nrg.2017.4

43. Wakefield JC (2016) Diagnostic Issues and Controversies in DSM-5: Return of the False Positives Problem. Annu Rev Clin Psychol 12:105-132. doi:10.1146/annurev-clinpsy-032814-112800

44. Wang X, HuangFu C, Zhu X, Liu J, Gong X, Pan Q, Ma X (2021) Exosomes and Exosomal MicroRNAs in Age-Associated Stroke. Curr Vasc Pharmacol. doi:10.2174/1570161119666210208202621 
45. Weiss LA, Arking DE, Autism H, Daly C, Chakravarti A (2009) A genome-wide linkage and association scan reveals novel loci for autism. Nature, 461(7265), 802-808. doi:10.1038/nature08490

46. Yang J, Chen Y, Xiong X, Zhou X, Han L, Ni L, Huang C (2018) Peptidome Analysis Reveals Novel Serum Biomarkers for Children with Autism Spectrum Disorder in China. Proteomics Clin Appl 12(5):e1700164. doi:10.1002/prca.201700164

47. Yao F, Zhang KY, Feng CY, Gao Y, Shen LM, Liu XK, Ni JZ (2021) Protein Biomarkers of Autism Spectrum Disorder Identified by Computational and Experimental Methods. Frontiers in Psychiatry, 12. doi:ARTN 554621 3389/fpsyt.2021.554621

48. Yu TW, Chahrour MH, Coulter ME, Jiralerspong S, Okamura-lkeda K, Ataman B, Walsh CA (2013) Using Whole-Exome Sequencing to Identify Inherited Causes of Autism. Neuron 77(2):259-273. doi:10.1016/j.neuron.2012.11.002

\section{Figures}

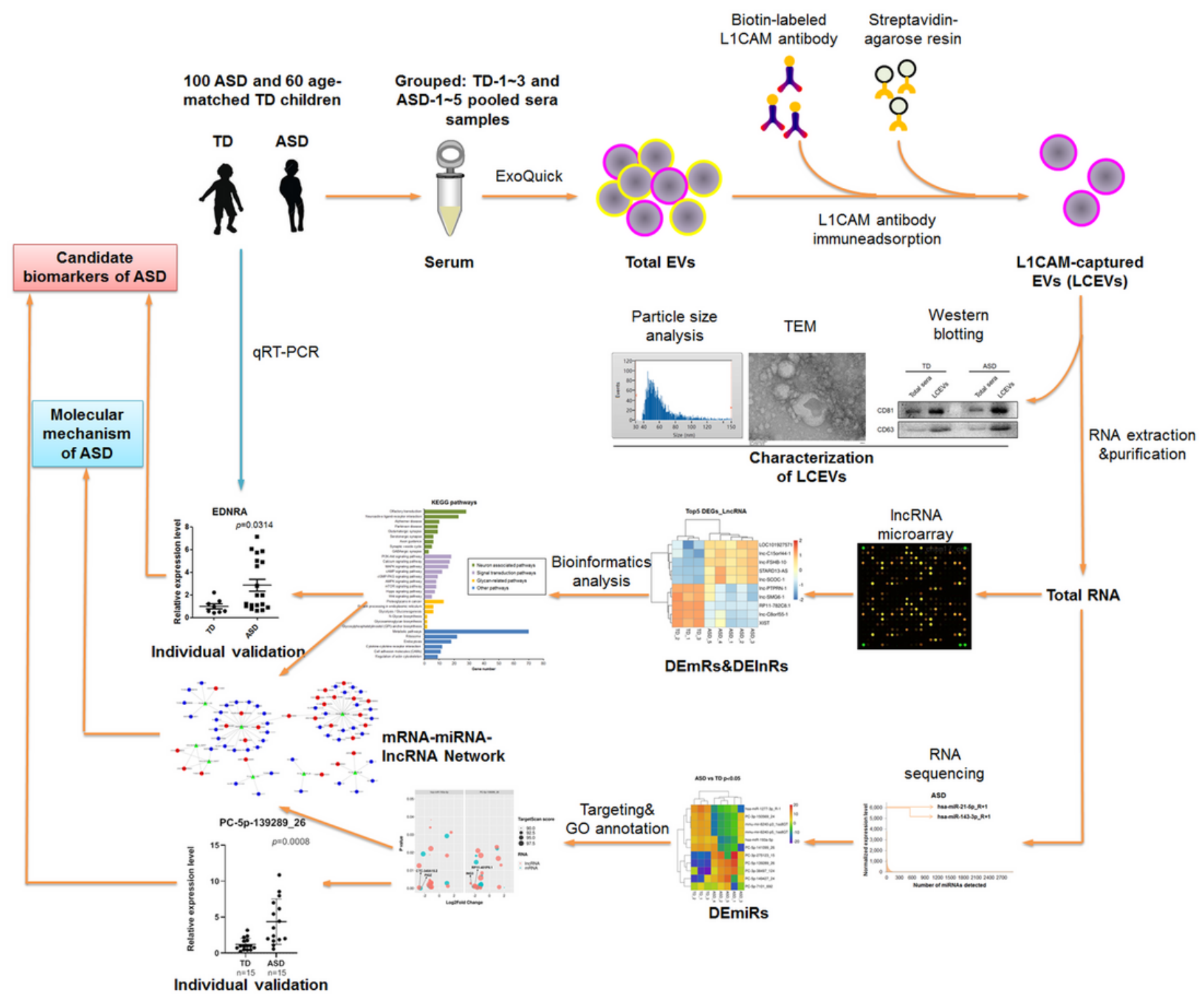


Figure 1

Schematic flow diagram of the integrated strategy used herein.

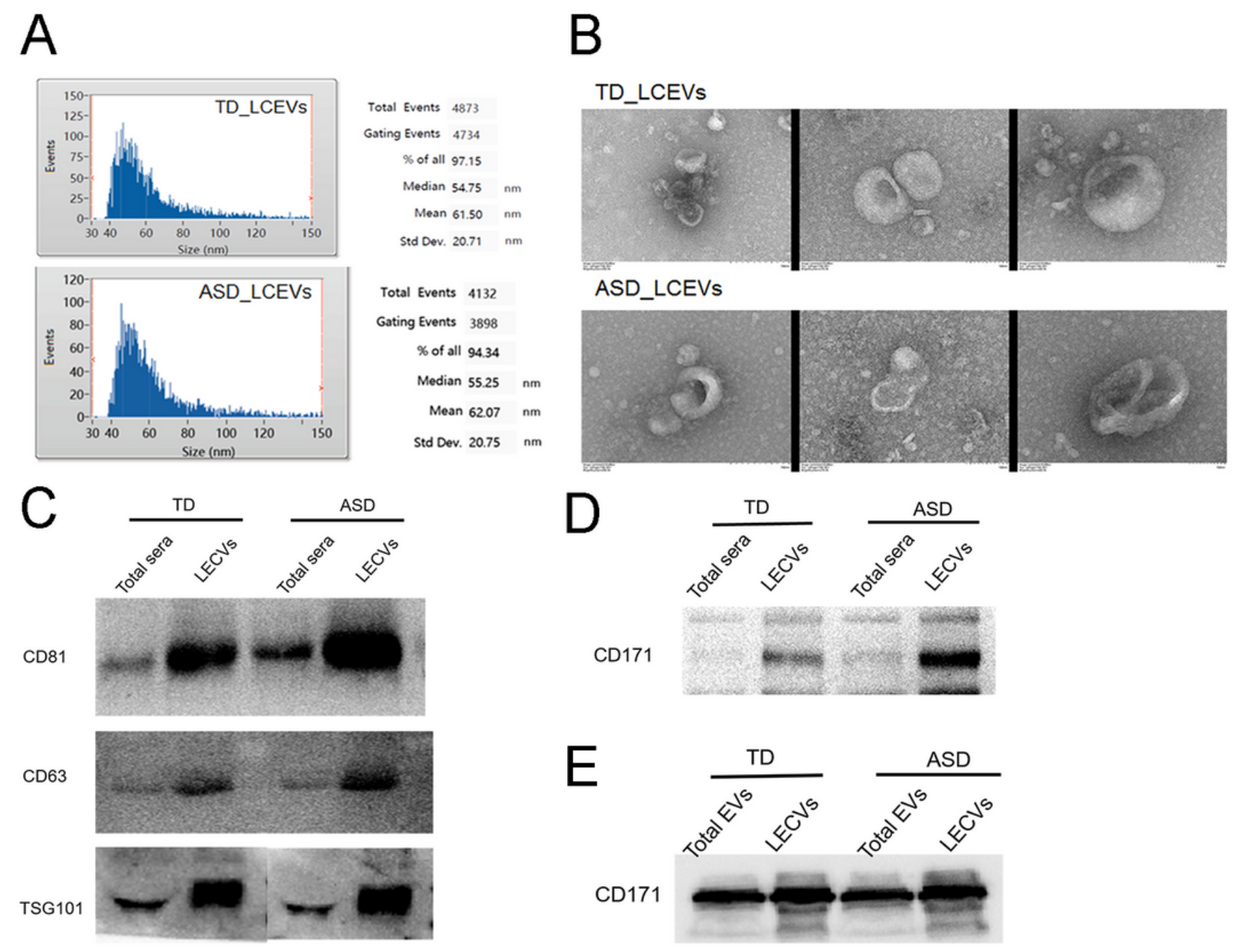

Figure 2

Characterization of the serum LCEVs. A. Nanoparticle tracking analysis of LCEVs in the ASD group and the TD group. B. The image of LCEVs under transmission electron microscopy. C. WB analysis of EVspecific marker proteins CD63 and CD81 in serum and the obtained LCEVs solution. D. WB analysis of the expression of L1CAM in the total serum EVs and in the LCEVs. 
A

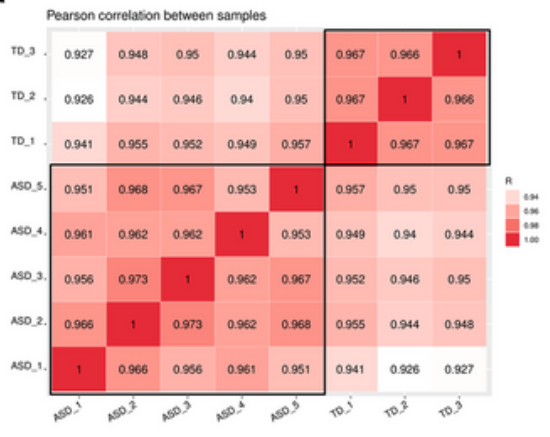

B

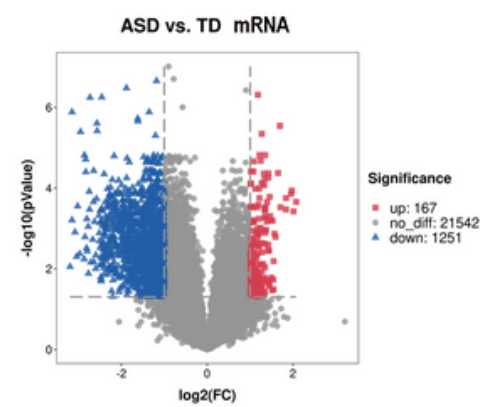

C

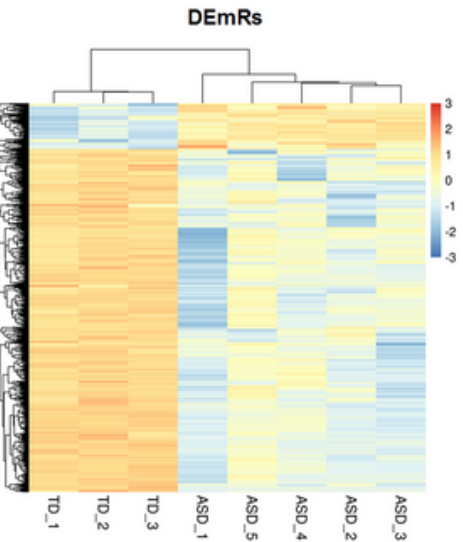

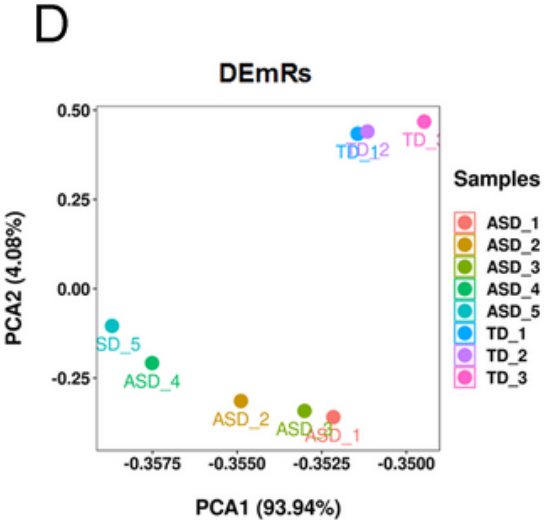

F $\square$ biological_process $\square$ cellular_component $\square$ molecular_function

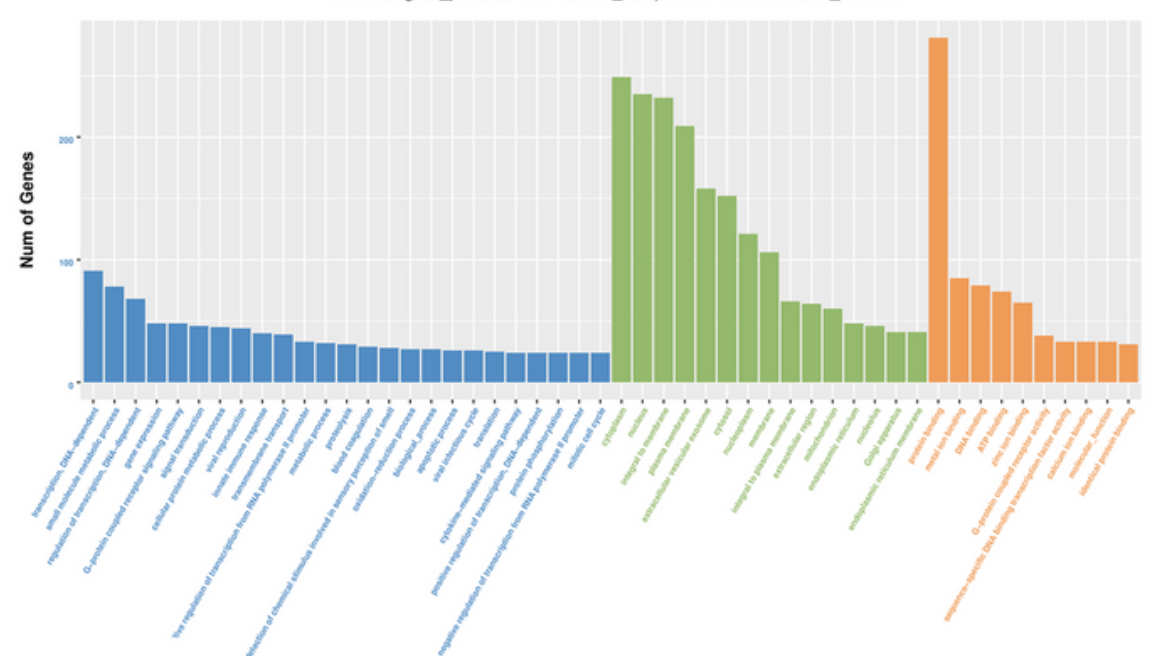

GO_term

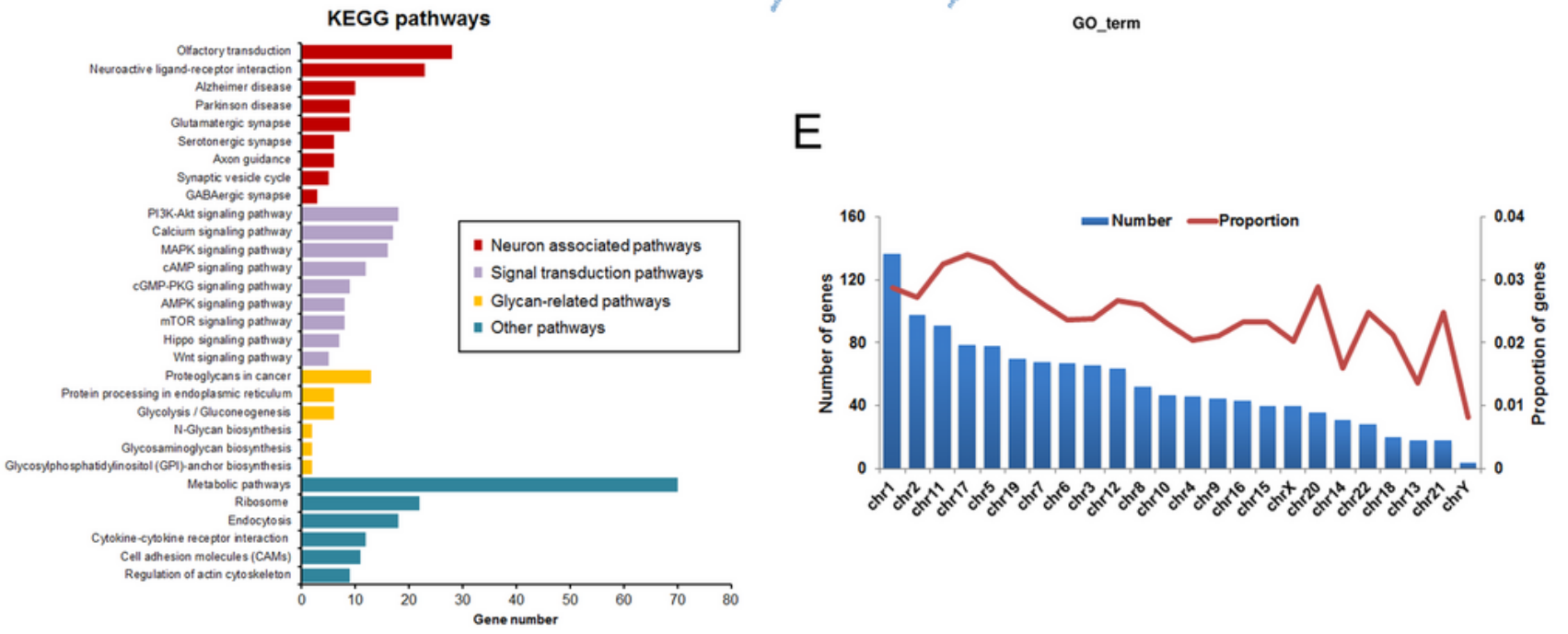

Figure 3

Differential expression and bioinformatic analysis of mRNAs in ASD serum LCEVs. A. Pearson's correlation analysis of all RNAs among different subgroups based upon IncRNA microarray. B. The differentially expressed mRNAs (DEmRs) with at least twofold change and a $P$ value of less than 0.05 in ASD. C. Hierarchical clustering analysis (HCA) of the DEmRs in subgroups of TD-1-3 and ASD-1-5.The subgroups were listed in columns, and DEmRs were listed in rows. The color and intensity of each square 
indicates expression levels relative to the other data in the row. Red, high; blue, low; white, medium. D. Principal component analysis (PCA) of DEmRs in subgroups of TD-1-3 and ASD-1-5. The color of each circle indicated a subgroup. E. The number and proportion of genes for DEmRs distributed on chromosomes. GO annotation (F) and KEGG analysis (G) of the DEmRs in ASD.

A

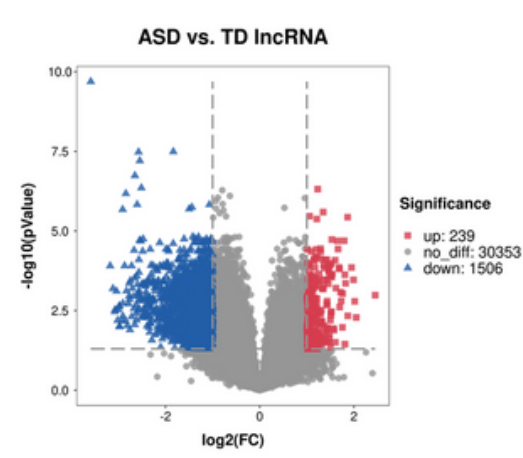

D

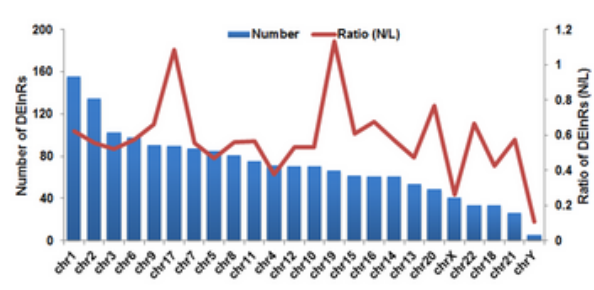

G

Cellular component
B

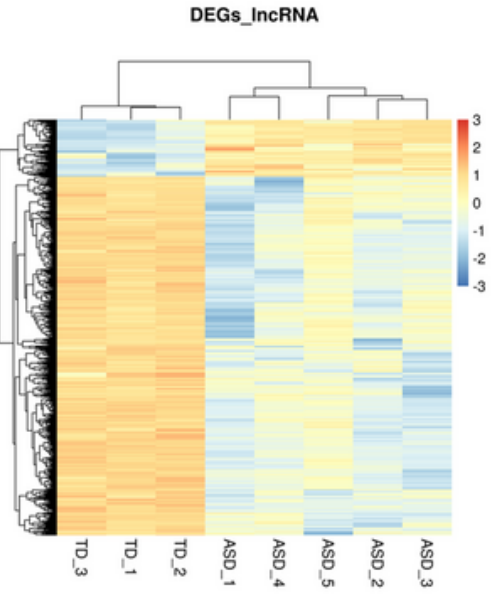

$E$

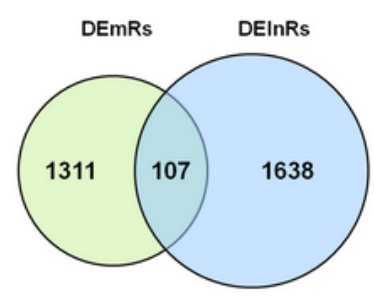

$\mathrm{H}$
C
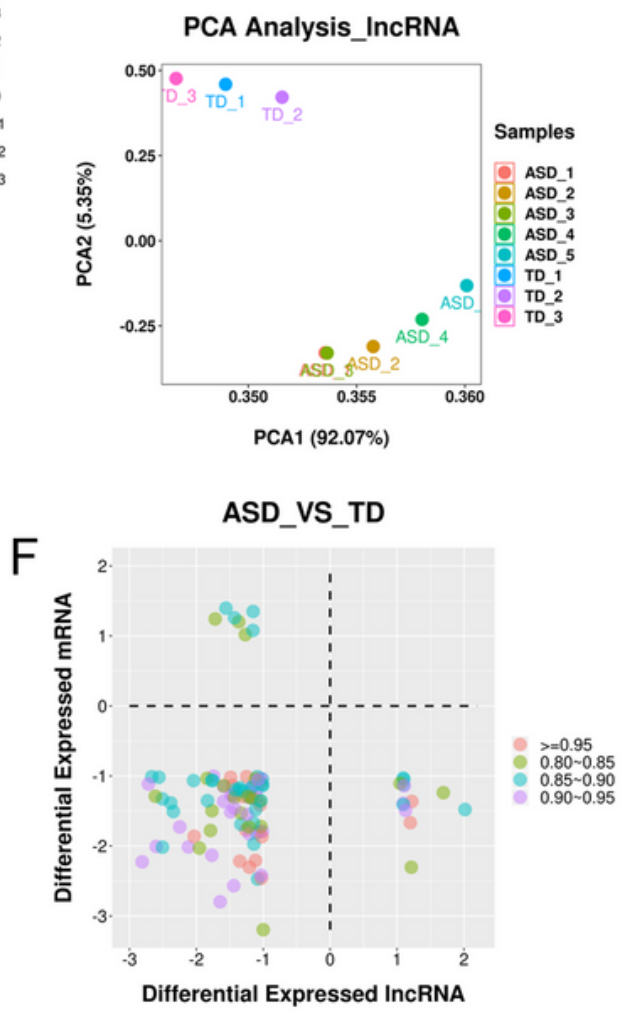

Molecular function
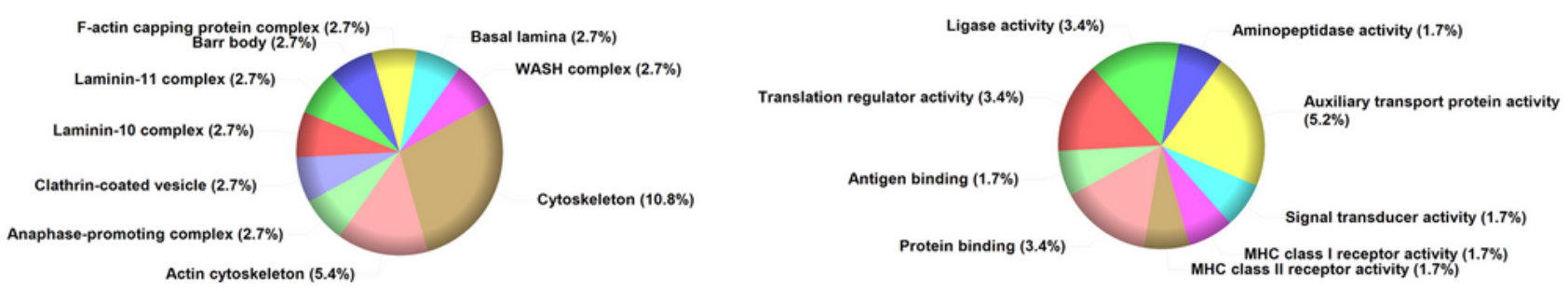

I

Biological process

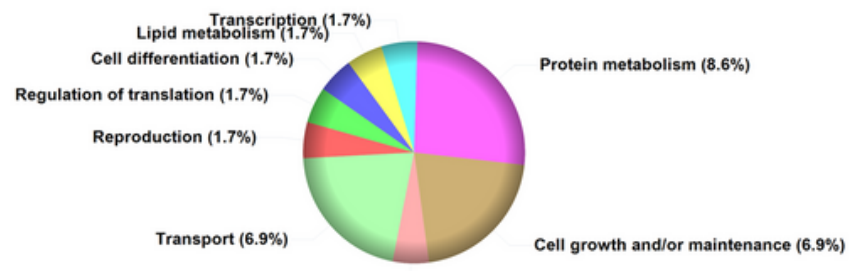

Apoptosis (1.7\%)

Figure 4 
Differential expression and functional prediction of IncRNAs in ASD serum LCEVs. A. The differentially expressed IncRNAs (DElnRs) with at least twofold change and a P value of less than 0.05 in ASD. B. HCA of the DEmRs in subgroups of TD-1-3 and ASD-1-5. The subgroups were listed in columns, and DElnRs were listed in rows. The color and intensity of each square indicates expression levels relative to the other data in the row. Red, high; blue, low; white, medium. C. PCA of DEmRs in subgroups of TD-1-3 and ASD-15. The color of each circle indicated a subgroup. D. The number of DElnRs on each chromosome and the ratio of the number of DEInRs to the length of that chromosome. E. Venn diagram of the DEmRs and DElnRs and their targeting relationship (overlapped). F. Double-omic analysis of the 107 pairs of DElnRDEmR. The color of the dot indicated correlation coefficient. The axis represented log2 fold change (ASD/TD). GO annotation of target genes for DElnRs according to the three grouping classifications of cellular component $(G)$, molecular function $(H)$ and biological process $(I)$. 
A

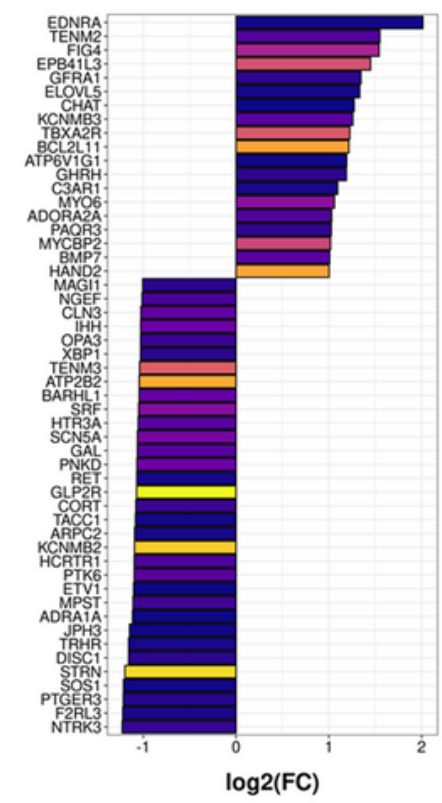

C

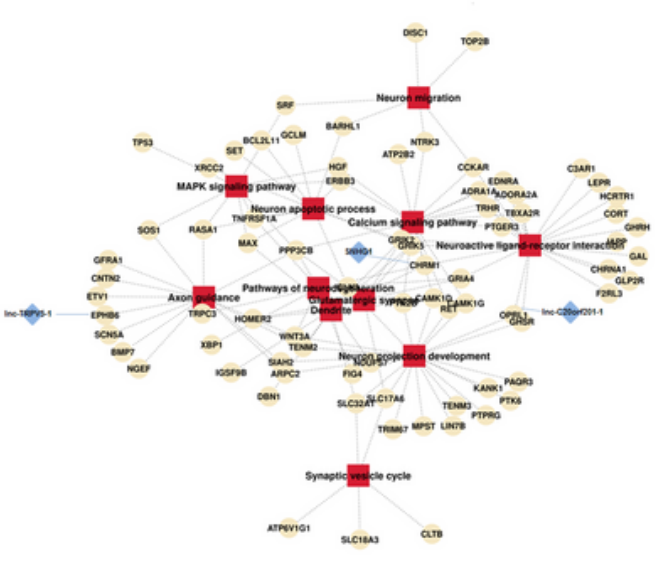

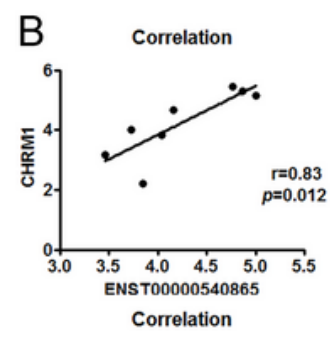

p Value

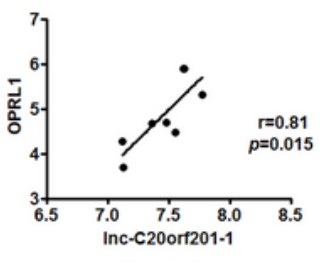

Correlation

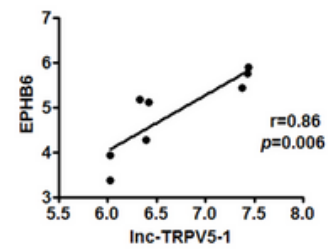

D

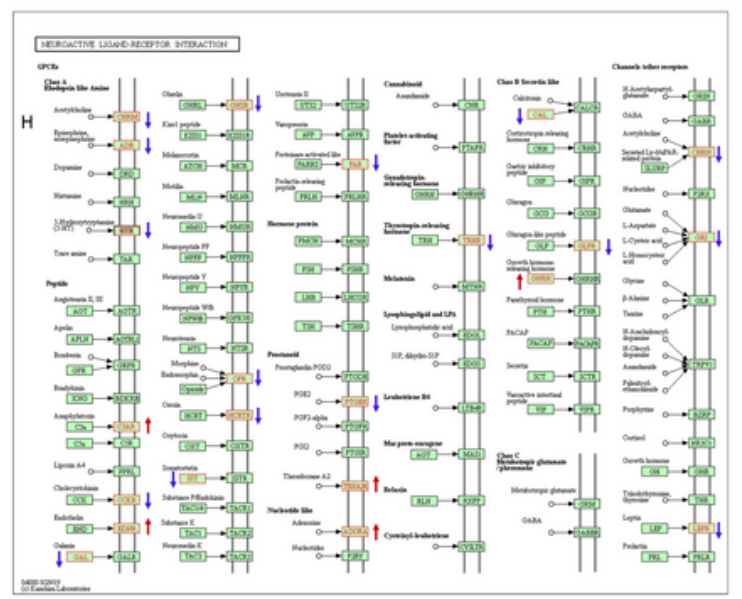

E

EDNRA

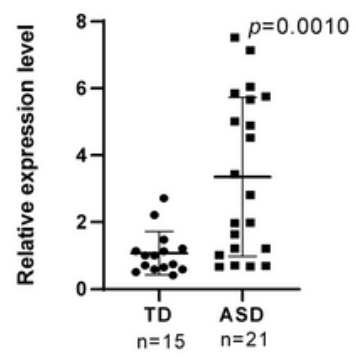

SLC17A6

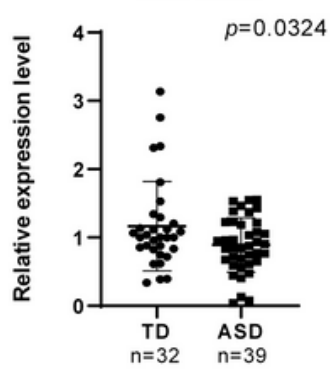

HTR3A

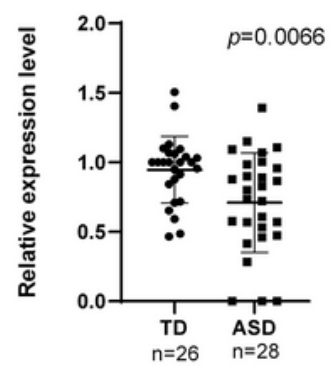

\section{Figure 5}

Neuron-related network and significant markers in ASD. A. The $\log _{2}$ foldchanges and $p$ values of 104 DEmRs that were related to neuron in ASD. B. The positive relationship of IncRNAs SNHG1, IncC20orf201-1 and Inc-TRPV5-1 with their target genesCHRM1, OPRL1 and EPHB6, respectively. The r value and $p$ value was calculated. The $p$ value was lower than 0.05 . C. Neuron-related network associated with ASD according to GO and KEGG analyses. The red square represented neuron related functions; the 
yellow circle represented DEmRs; and the blue diamond represented DElnRs. D. The most enriched pathway, neuroactive ligand-receptor interaction, which contained 5 up-regulated mRNAs and 19 downregulated mRNAs in ASD. Red arrow represented up-regulated and blue arrow represented down-regulated in ASD. E. Individual validation of the differential expression of EDNRA, SLC17A6 and HTR3Aby qRT-PCR.

A

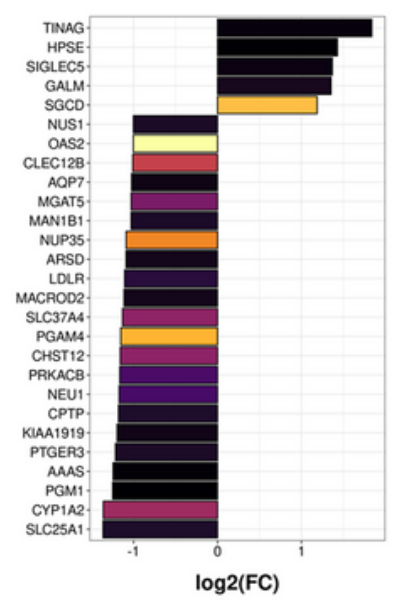

C

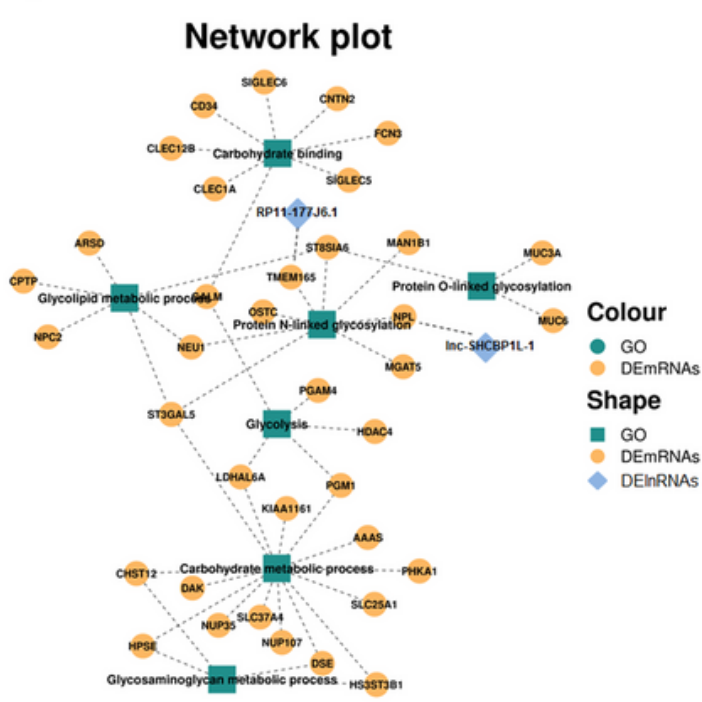

$E$

TMEM165

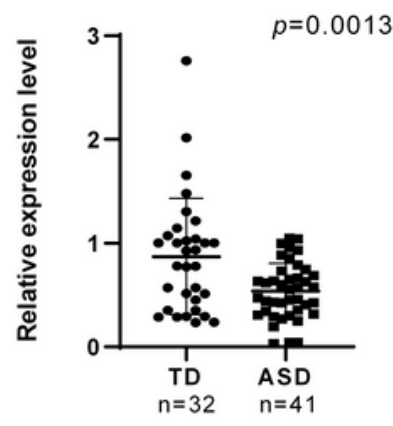

B
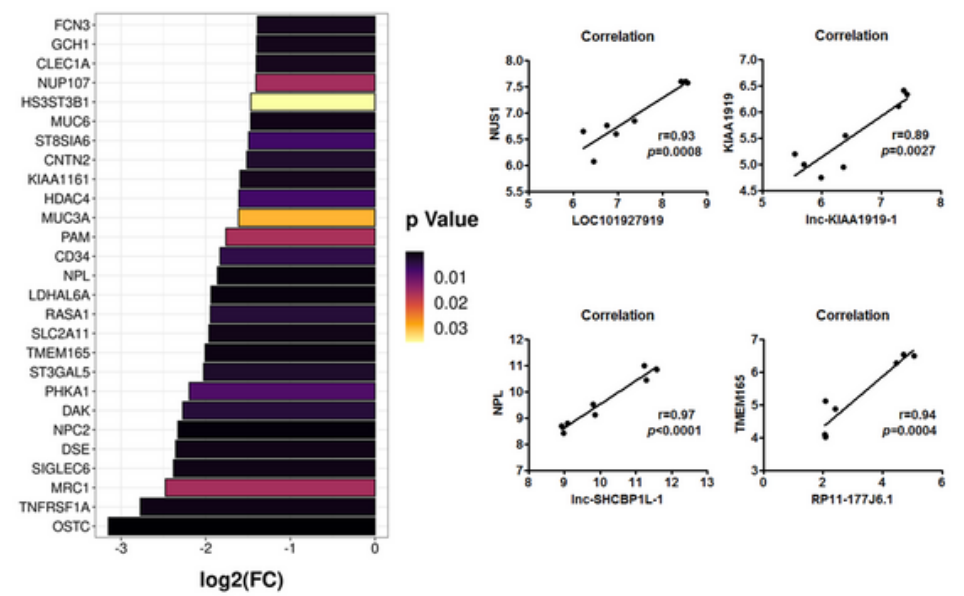

$\mathrm{D}$

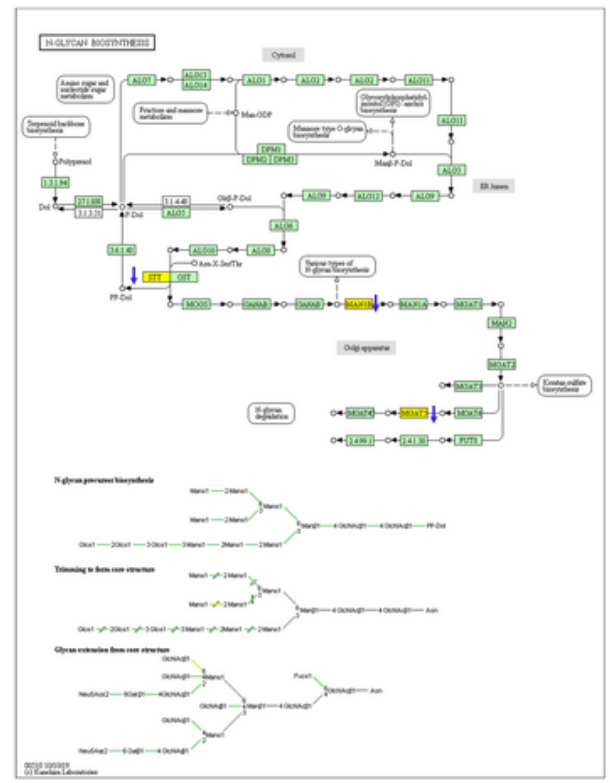

OSTC

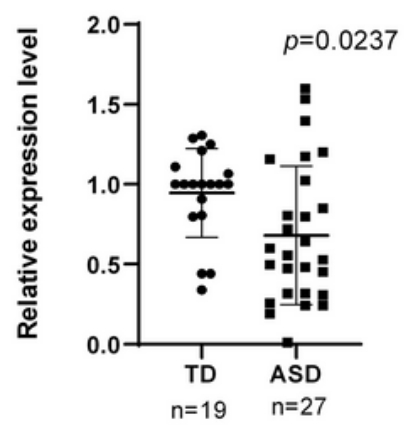

Figure 6 
Glycan-related network and candidate markers in ASD. A. The $\log _{2}$ foldchanges and $p$ values of 54 DEmRs that were related to glycan metabolism in ASD. B. The positive relationship of IncRNAs LOC101927919, Inc-KIAA1919-1, Inc-SHCBP1L-1 and RP11-177J6.1 with their target genes NUS1, KIAA1919, NPL and TMEM165, respectively. The $r$ value and $p$ value was calculated. The $p$ value was lower than 0.05. C. Glycan-related network associated with ASD according to GO and KEGG analyses. The green square represented carbohydrate associated functions; the orange circle represented DEmRs; and the blue diamond represented DElnRs. D. Expressions of OSTC, MAN1B1 and MGAT5 were downregulated in $\mathrm{N}$-glycan biosynthesis implicated in ASD. Blue arrow represented down-regulated in ASD. E. Individual validation of the differential expression of OSTC and TMEM165 by qRT-PCR. 
B

TD

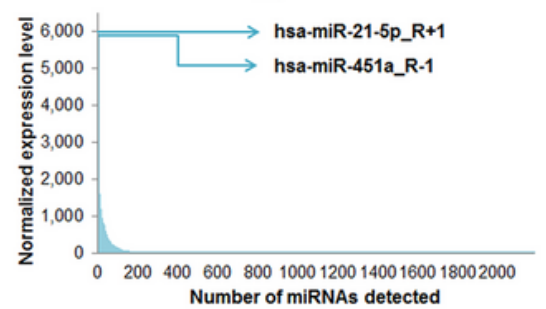

\section{C}

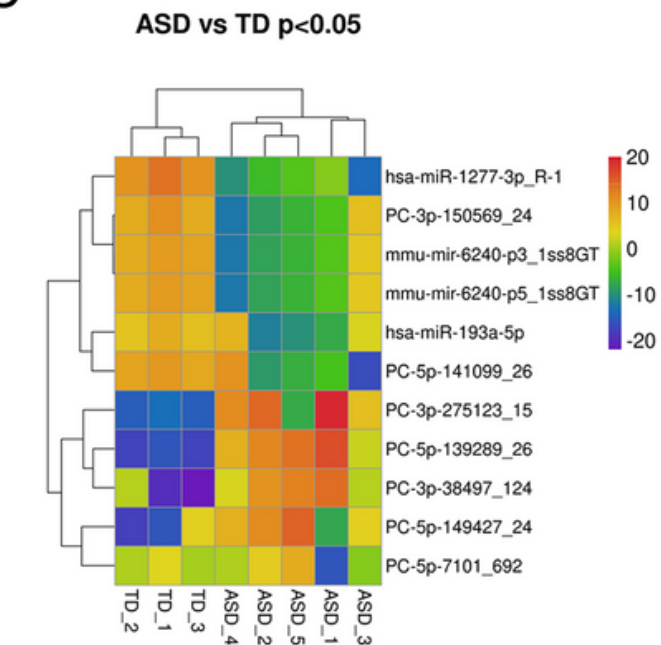

D

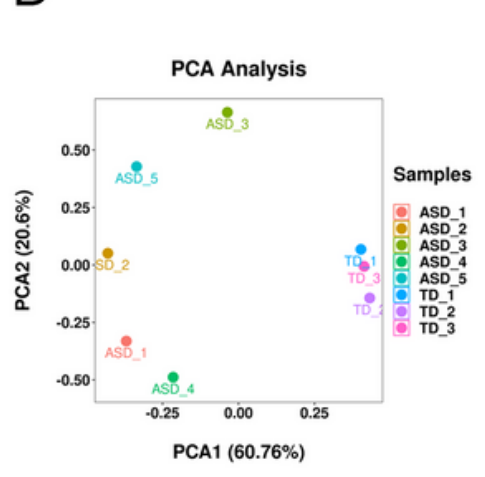

DEmiRNAs

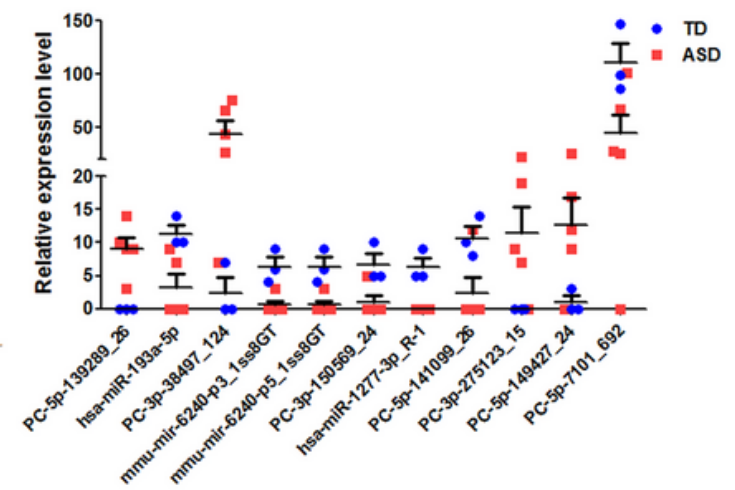

E

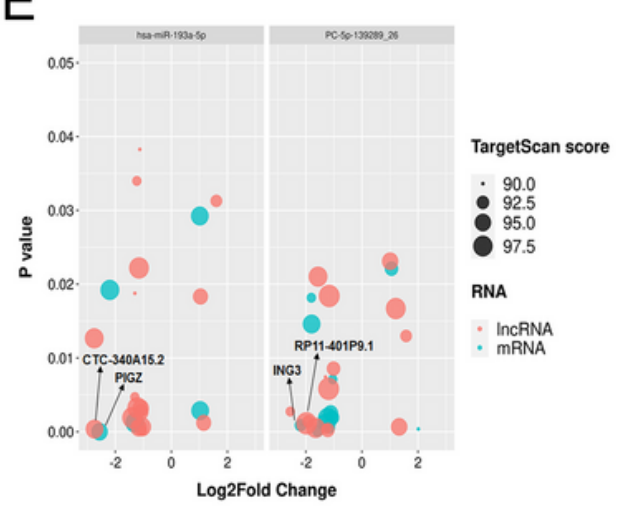

F

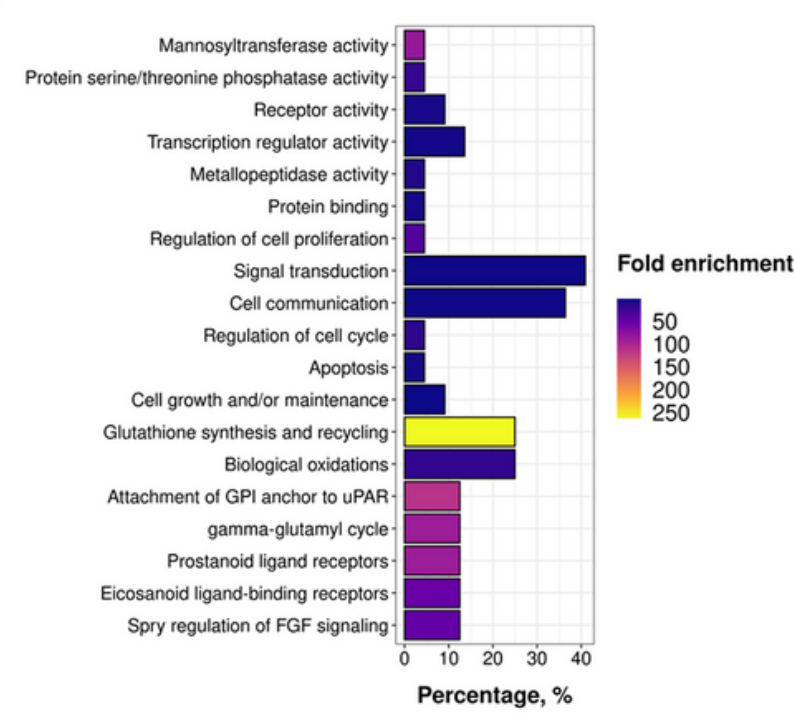

G

PC-5p-139289_26

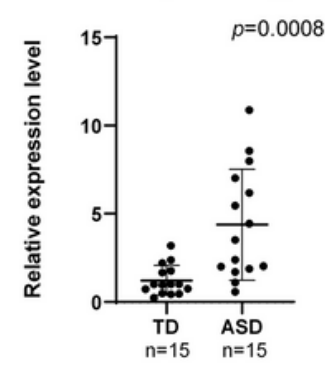

hsa-miR-193a-5p

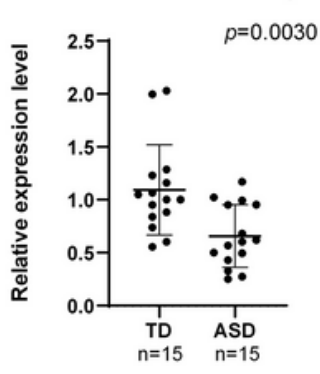

\section{Figure 7}

Differential expression of miRNA in ASD serum LCEVs. A. Abundance of the miRNAs in TD and ASD. The normalized expression level of each miRNAs was represented as the mean of the read counts in biological replicates. B. The normalized expression level of 11 DEmiRs in subgroups of TD and ASD. C. Eleven miRNAs were differentially expressed across subgroups. The 11 DEmiRs $(p<0.05)$ across ASD (ASD_1-5) and TD (TD_1-3) subgroups are clustered in the heatmap using a complete clustering 
algorithm. D. PCA of DEmiRs in subgroups of TD-1-3 and ASD-1-5. The color of each circle indicated a subgroup. E. Two miRNAs, namely PC-5p-139289_26 and hsa-miR-193a-5p, had the largest number of predicted targets with high confidence (TargetScan score>90). F. GO annotation revealed the percentages of genes and the fold enrichments for all cellular activities. G. Individual validation of the differential expression of PC-5p-139289_26 and hsa-miR-193a-5p by qRT-PCR.

\section{Supplementary Files}

This is a list of supplementary files associated with this preprint. Click to download.

- Supplementaryfigures.docx

- Supplementarytables.xlsx 